Article

\title{
A General and Scalable Synthesis of Polysubstituted Indoles
}

\author{
David Tejedor ${ }^{1, *}$, Raquel Diana-Rivero ${ }^{1,2}$ and Fernando García-Tellado ${ }^{1, * \mathbb{D}}$ \\ 1 Instituto de Productos Naturales y Agrobiología, Consejo Superior de Investigaciones Científicas, \\ Astrofísico Francisco Sánchez 3, 38206 La Laguna, Spain; raquel.diana.rivero@gmail.com \\ 2 Doctoral and Postgraduate School, Universidad de La Laguna, Apartado Postal 456, 38200 La Laguna, Spain \\ * Correspondence: dtejedor@ipna.csic.es (D.T.); fgarcia@ipna.csic.es (F.G.-T.)
}

Received: 3 November 2020; Accepted: 26 November 2020; Published: 28 November 2020

check for updates

\begin{abstract}
A consecutive 2-step synthesis of $N$-unprotected polysubstituted indoles bearing an electron-withdrawing group at the C-3 position from readily available nitroarenes is reported. The protocol is based on the [3,3]-sigmatropic rearrangement of $\mathrm{N}$-oxyenamines generated by the DABCO-catalyzed reaction of $\mathrm{N}$-arylhydroxylamines and conjugated terminal alkynes, and delivers indoles endowed with a wide array of substitution patterns and topologies.
\end{abstract}

Keywords: indol; indole-3-carboxylates; indole-3-carboxyamides; 3-aroylindoles; 3-acylindoles; $\mathrm{N}$-oxyenamines; [3,3]-sigmatropic rearrangement; annulation; DABCO; organocatalysis

\section{Introduction}

The indole moiety occupies a privileged place in the realm of heterocyclic chemistry [1]. It constitutes the core structure of many natural products and synthetic bioactive compounds exhibiting a wide array of biological activities [2-6], and has also found use as structural parts of material science devices [7-9] and chiral catalysts [10-12]. Recent studies have shown that the substitution at the C-3 position with electron-withdrawing groups (3-EWG-indoles) not only endows these structures with important pharmaceutical/therapeutic activities [13-21], but also it converts them into versatile synthetic molecular platforms in heterocyclic chemistry, such as, in dearomatization-based synthetic disconnections [22]. The catalytic asymmetric dearomatization of 3-nitroindoles constitutes an iconic example of this synthetic potential [23-34]. The most traditional synthetic approach to these functionalized indoles relies on the Friedel-Crafts acylation of preformed indoles [35-37]. This transformation suffers important drawbacks such as mandatory nitrogen protection, regioselectivity control, stoichiometric use of metal salts and Lewis acid promoters, and strict exclusion of moisture. Although modern alternatives using metal-catalysis [38-42] or photocatalysis [43-46] have overcome some of these difficulties, the implementation of real-world strategies to prepare these heterocycles from simple and widely accessible starting materials remains a synthetic challenge in medicinal and synthetic chemistry. In spite of the many advances achieved in the synthesis of indoles during the last 100 years [47], the de novo synthesis of 3-EWG-indoles remains largely unexplored. A large number of strategies for the synthesis of indoles use alkynes and a convenient nitrogen source as substrates [48] and the annelation of the five-membered ring to an existing functionalized benzene ring as the main synthetic disconnection [49]. The inherent alkyne reactivity and the broad access to nitrogen sources constitute practical advantages for these strategies. Among them, the [3,3]-sigmatropic rearrangement of $N$-oxyenamines [50] or 1-aryl-2-vinylhydrazines [51,52] have been objects of profuse research (Scheme 1a). Prototypical examples of these strategies constitute the Bartoli [53,54] (N-oxyenamines) and the Fischer [55] (1-aryl-2-vinylhydrazines) syntheses of indoles. Although both reactions have been largely used in the preparation of indoles [47], they present 
important drawbacks for general preparative synthetic use. Whereas those based on the Fischer disconnection suffer the limitations associated with the hydrazine synthesis and drastic reaction conditions, the Bartoli method, although synthetically powerful, requires the use of ortho-substituted nitroarenes and functional tolerance to Grignard reagents. Recently, an elegant example of the use of the $N$-oxyenamine disconnection to gain access to polysusbtituted indoles has been reported by Zhang and co-workers (Scheme 1b) [56]. The methodology uses the Zn/Au-catalyzed reaction of N-protected $N$-arylhydroxamic acids (or $N$-aryl- $N$-hydroxycarbamates) $\mathbf{1}$ and alkynes to construct the indole core with a diverse pattern of substitution at both rings. Although the methodology was effective for the synthesis of alkyl indole-3-carboxylates (2, the unique reported example: $\mathrm{Pg}=\mathrm{Ac} ; \mathrm{R}^{1}=\mathrm{CH}_{2} \mathrm{CH}_{2} \mathrm{Ph}_{\text {, }}$ $\mathrm{R}^{2}=\mathrm{CO}_{2} \mathrm{Me}$ ), its extension to other 3-EWG-indoles remains to be assessed [57].

a General [3,3]sigmatropic disconnection.

CC bond formation Cyclization/aromatization

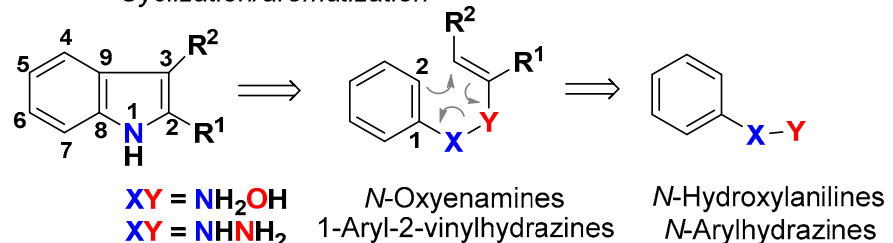

b Zn/Au-catalyzed synthesis of substituted indoles.

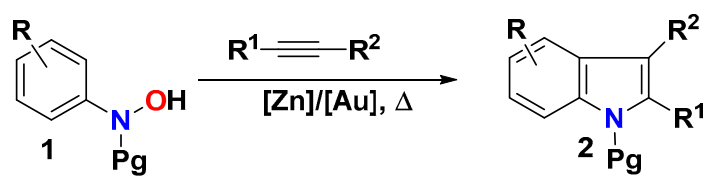

c This work: consecutive 2-step reaction manifold.
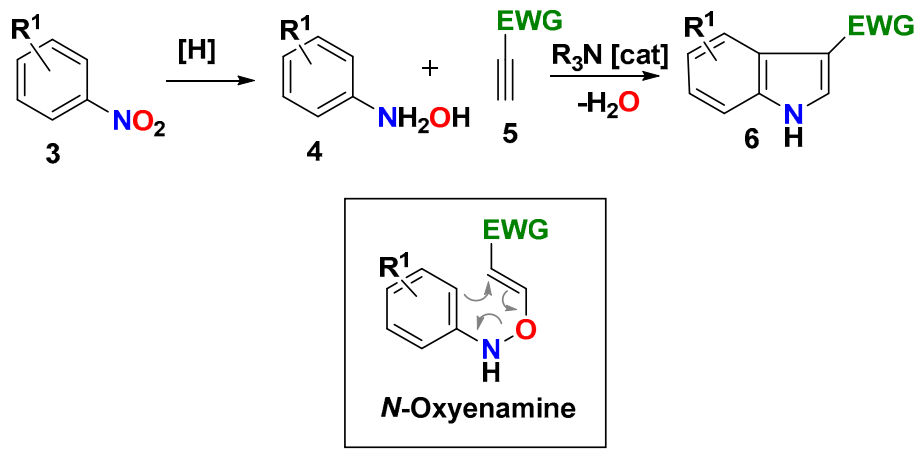

Scheme 1. Strategy for the synthesis of indoles 6 with electron-withdrawing group at the C-3 position (3-EWG indoles). (a) General [3,3] sigmatropic disconnection. (b) Zn/Au-catalyzed synthesis of indoles.

(c) This work: consecutive two-step reaction manifold.

We envisioned that a simpler and general methodology using the $N$-oxyenamine disconnection could be arranged on the basis of the well-established addition of nucleophiles onto terminal conjugated alkynes catalyzed by tertiary amines (Scheme 1c) [58-61]. When searching for precedents for this disconnection, we found a reduced number of reported examples, all of them circumscribed to the synthesis of alkyl indole-3-carboxyates [62-66], and with important drawbacks for a practical and general application. They mostly use $N$-arylhydroxylamine salts or $N$-protected $N$-arylhydroxylamines to force the $O$-addition reaction, and require large excesses of alkyne and long reaction times to deliver the indole-3-carboxylates in low to moderate yields. Therefore, a general and practical protocol for accessing NH-free 3-EWG-indoles based on this disconnection remains to be established. In terms of sustainability, such protocol should be direct, operationally simple and scalable, avoiding non-productive $\mathrm{N}$-protection/N-deprotection steps. We report herein our results on the implementation 
of a strategy fulfilling these requirements and overcoming the practical shortcomings found in previous reports. The strategy delivers NH-free multisubstituted 3-EWG-indoles through a consecutive 2-step reaction manifold, and it uses nitroarenes and activated terminal alkynes as building blocks (Scheme 1c). The reaction manifold takes advantage of the $O$-reactivity of $N$-arylhydroxylamines toward terminal conjugated alkynes and of the rich arsenal of commercially available functionalized nitroarenes. Whereas the first one avoids unnecessary $\mathrm{N}$-protection/ $\mathrm{N}$-deprotection steps (step-economy, reagent-economy), the second one offers an almost countless catalogue of functional diversity on the benzene ring (general outcome). These properties make this reaction manifold a convenient, instrumentally simple, robust and reliable synthetic tool for the exploration/annotation of the chemical space of 3-EWG-indoles.

\section{Results and Discussion}

\subsection{Initial Experiments and Optimization of the Reaction Conditions for a Consecutive 2-Step Protocol}

The initial studies began using commercially available $N$-benzoyl- $N$-phenylhydroxylamine $(4 \mathbf{a}-\mathbf{N}-\mathbf{B z})$ as the nitrogen species needed for the synthesis of indoles and the standard conditions reported by our group for the DABCO-catalyzed synthesis of propargyl vinyl ethers [59] (Scheme 2). Although the addition of the hydroxylamine to methyl propiolate (5a) took place in the presence of DABCO, and this was followed by the desired cyclization, the hydroxyindoline intermediate HI was isolated instead of the expected indole 6a-N-Bz. Furthermore, a side-product incorporating a second unit of methyl propiolate was also obtained (DVE). It forms from the DABCO-catalyzed addition of methyl propiolate (5a) on the enol ether generated on the [3,3]sigmatropic rearrangement of the intermediate $\mathrm{N}$-oxyenamine. The overnight reaction of intermediate $\mathrm{HI}$ with $\mathrm{Et}_{3} \mathrm{~N}$ afforded the indole 6a-N-Bz in quantitative yield.

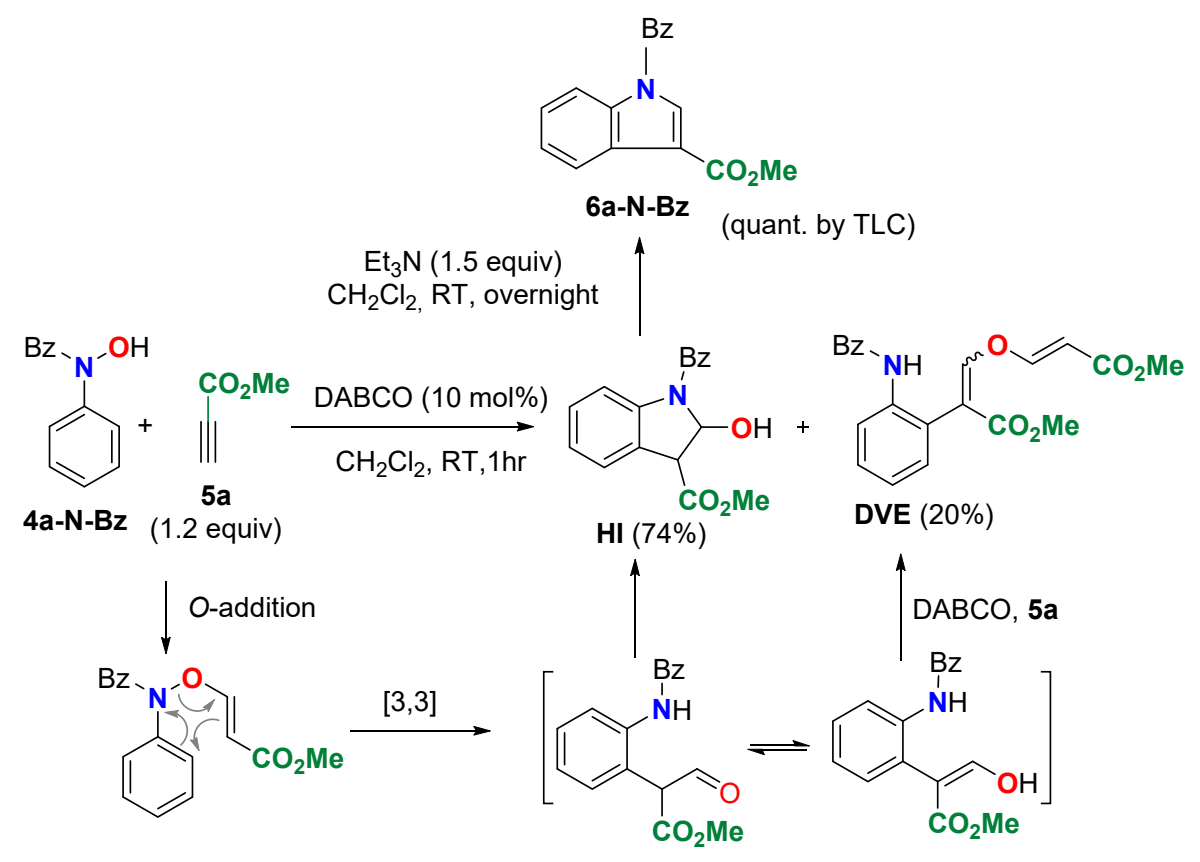

Scheme 2. DABCO-catalyzed reaction of N-Benzoyl-N-phenylhydroxylamine (4a-N-Bz) and methyl propiolate (5a).

At this time, we decided to question the need for the protecting group on the nitrogen atom. Indeed, when starting from $N$-phenylhydroxylamine (4a) and methyl propiolate (5a) (1.2 equiv) as the reactants, the reaction proceeded smoothly to the desired unprotected indole 6 a (Table 1, entry 1). The observed outcome was different to that reported by Hwu et al. [64] in which $N$-vinyl protected indoles were 
obtained when $N$-phenylhydroxylamine (4a) reacted with an excess of methyl propiolate (5a) in the presence of a catalytic amount of DMAP. Strikingly, the report by Hwu et al. claims that DABCO and $\mathrm{Et}_{3} \mathrm{~N}$ did not give significant amounts of the desired indoles under their reaction conditions.

Table 1. Optimization of the reaction conditions.

$\mathrm{H}_{\mathbf{N}^{-}} \mathrm{OH}$

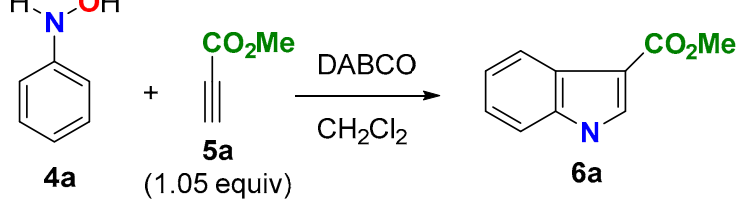

\begin{tabular}{ccccc}
\hline entry & DBACO & Temperature & Time & Yield (6a) \\
\hline 1 & $10 \mathrm{~mol} \%$ & RT & $1 \mathrm{~h}$ & $68 \%$ \\
2 & $10 \mathrm{~mol} \%$ & $0{ }^{\circ} \mathrm{C}$ to RT & $1 \mathrm{~h}$ & $83 \%$ \\
3 & $1 \mathrm{~mol} \%$ & $0{ }^{\circ} \mathrm{C}$ to RT & Overnight & $75 \%$ \\
4 & $5 \mathrm{~mol} \%$ & $0{ }^{\circ} \mathrm{C}$ to RT & $1 \mathrm{~h}$ & $82 \%$ \\
5 & $5 \mathrm{~mol} \%$ & $-25{ }^{\circ} \mathrm{C}$ to RT & $2 \mathrm{~h}$ & $86 \%$ \\
\hline
\end{tabular}

Red: To highlight that these are the best conditions.

Once the feasibility of the reaction using free $N$-phenylhydroxylamine (4a) was established, we next undertook the optimization of the reaction (Table 1). Under all assayed conditions, the formation of the desired indole 6a was accompanied by the formation of very small amounts of side-product DVE. Based on these results, and taking into consideration the simplicity of the experimental procedure, we chose the reaction conditions depicted in entry 4 as the standardized conditions for the formation of the desired indoles 6 from the appropriate $N$-arylhydroxylamines 4 .

Once this reaction was optimized, we coupled this reaction with the reduction of the nitrobenzene (3a) to set up a consecutive 2-step reaction manifold (Scheme 3). Thus, reduction of nitrobenzene (3a) with hydrazine monohydrate and Rh on activated charcoal $(5 \%)$ in THF gave $N$-phenylhydroxylamine (4a), which, after filtration of the reaction mixture and concentration, was directly submitted to the next reaction step to deliver indole $6 \mathrm{a}$ in $80 \%$ overall yield. Under these conditions, the overall yield of indole 6 a was comparable to that obtained directly from 4 a ( $80 \%$ vs $82 \%)$.

Consecutive 2-step reaction

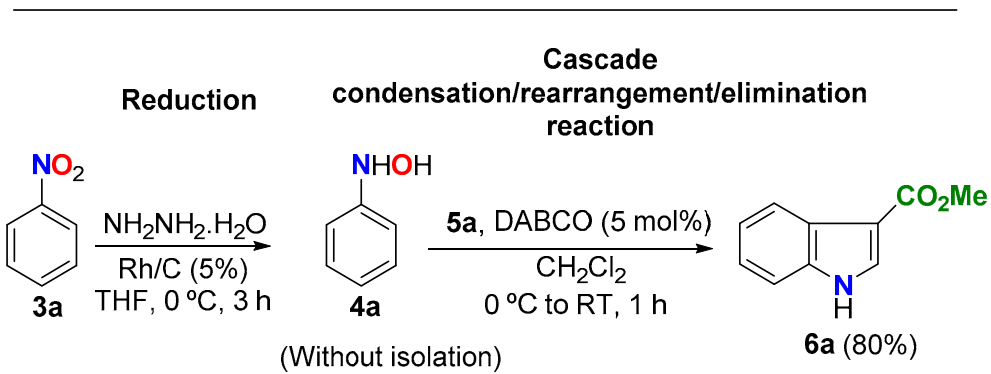

Scheme 3. Coupling reduction and cascade condensation/rearrangement/elimination reactions in a consecutive 2-step protocol.

\subsection{Scope of the Consecutive 2-Step Protocol}

With a convenient protocol in hand, we next explored its scope with regard to the alkyne using $N$-phenylhydroxylamine (4a) as the nitrogen component (Scheme 4). The reaction was well suited to prepare 3-EWG-indoles incorporating carboxylates (6a-c), aroyl (acyl) groups (6d,e), or carboxamides $(\mathbf{6 f}, \mathbf{g})$. In the case of $N, N$-disubstituted propionamides $\mathbf{5 f}$ and $\mathbf{5 g}$, the poorest Michael acceptors of the series, the reaction needed more time (overnight) to deliver the corresponding $\mathrm{N}, \mathrm{N}$-disubstituted indole-3-carboxamides $6 \mathrm{f}$ and $\mathbf{6 g}$ in synthetically relevant $52 \%$ and $58 \%$ yields 
respectively. The efficiency and simplicity of this transformation are emphasized when compared with the current multistep methodologies to prepare these indole-3-carboxamides [67].

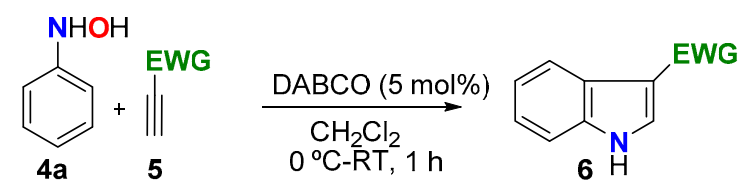

Esters<smiles>CCCCOC(=O)c1c[nH]c2ccccc12</smiles>

Ketones<smiles></smiles><smiles>CCCCC(=O)c1c[nH]c2ccccc12</smiles>

Amides<smiles>O=C(O)c1c[nH]c2ccccc12</smiles>

Scheme 4. Alkyne scope of the protocol. Reaction conditions: N-phenylhydroxylamine (4a) (4.0 mmol), alkyne 5 (4.2 mmol), DABCO (5 mol\%), $\mathrm{CH}_{2} \mathrm{Cl}_{2}(20 \mathrm{~mL}), 0{ }^{\circ} \mathrm{C}$ to $\mathrm{RT}, 1 \mathrm{~h}$. ${ }^{\text {a Overnight. }}$

The scope of the consecutive 2-step manifold with regard to the substitution at the benzene ring was explored using methyl propiolate (5a) as the alkyne component and differently substituted nitroarenes 3 (Scheme 5). The manifold tolerated a diverse substitution pattern on the benzene ring to deliver the corresponding methyl indole-3-carboxylates 6 with overall good efficiency and broad functional/structural diversity. A convenient grade of topological diversity could be generated, spanning from the simple monosubstituted derivatives (e.g., 6h-r, 52-90\% yield) to more complex polycyclic $(6 \mathbf{w}, 57 \%)$ or fused aromatic systems (6ac, 83\%). Meta-substituted $N$-arylhydroxylamines afforded separable mixtures of the corresponding C-4/C-6 regioisomers in excellent yields (80-90\%) and with a slight substituent- dependent regioselectivity (Scheme 5, compounds $\mathbf{6 n - r}$ ). A balance between steric and electronic effects of the substituent seems to steer the outcome of the [3,3]-sigmatropic rearrangement, in line with the reported results in the Fischer synthesis of indoles endowed with this substitution pattern $[52,65,68]$. Thus, electron-donating substituents $(\mathrm{R}=\mathrm{OMe})$ unbalanced the chemical outcome towards the less crowed isomer (6r, 1.7:1 / C-6: C-4 ratio) whereas electron-withdrawing groups did it in the opposite direction (6o-q; from 1:1 to 2.3:1 / C-4:C-6 ratio). It is remarkable that the [3,3]-sigmatropic rearrangement of benzyl ketene acetals bearing a meta-OMe substituent on the aromatic ring preponderantly delivers the C-4 isomer. Theoretical studies pointed to a stabilizing interaction between the lone pair of the substituent and the LUMO orbital of the transition state as being responsible for the observed regioselectivity [69]. The observed inverted regioselectivity in our reaction seems to rule out the existence of this kind of stabilizing interactions. 

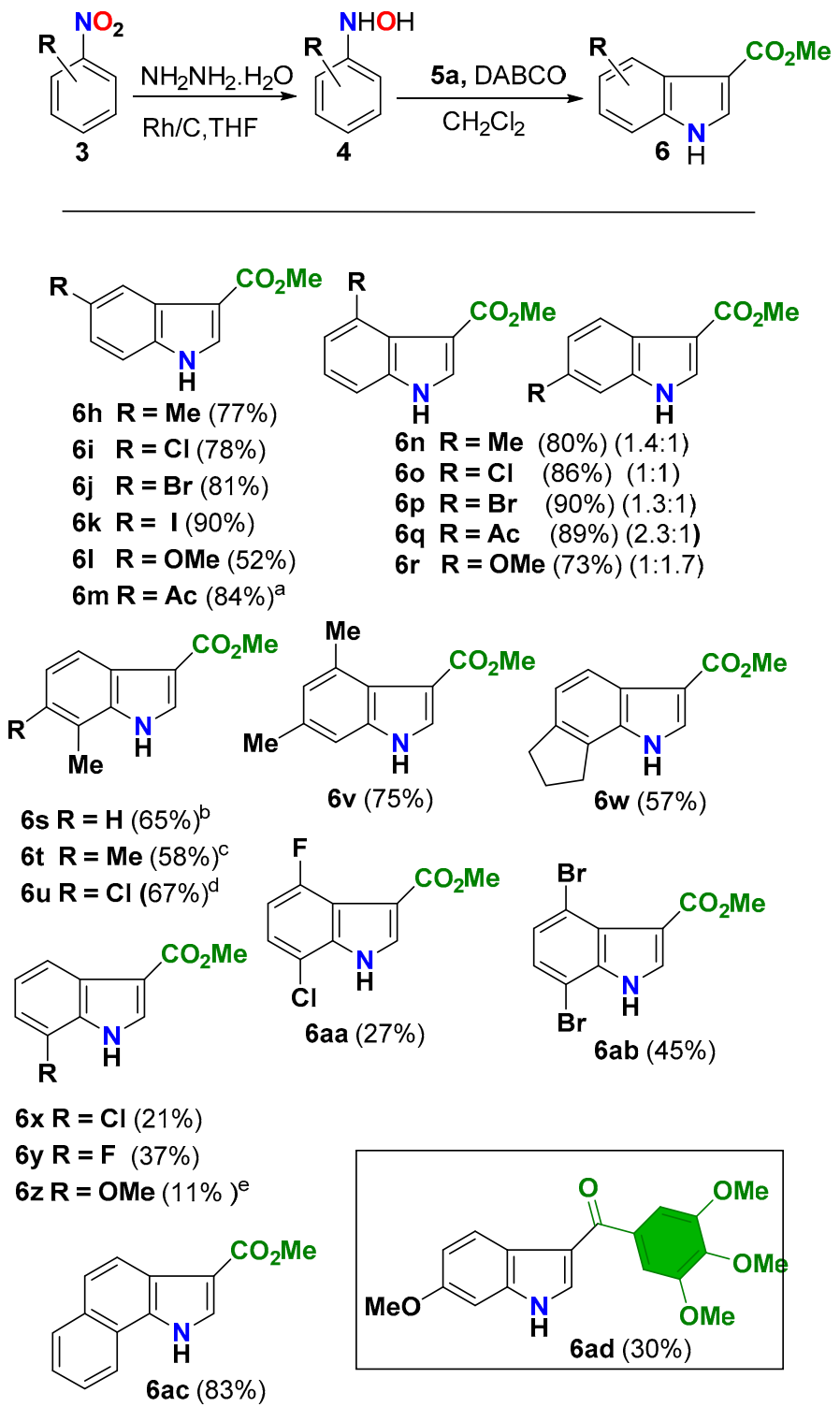

Scheme 5. Nitroarene scope of the protocol. Reaction conditions: 1) nitroarene 3 (4 mmol), $\mathrm{NH}_{2} \mathrm{NH}_{2} \cdot \mathrm{H}_{2} \mathrm{O}(4.4 \mathrm{mmol}), \mathrm{Rh} / \mathrm{C}(5 \%)(20 \mathrm{mg}), \mathrm{THF}(20 \mathrm{~mL}), 0{ }^{\circ} \mathrm{C}$ to $\mathrm{RT}, 3 \mathrm{~h}$, filtration on celite plug and concentration; 2) methyl propiolate (5a) (4.2 mmol), DABCO (5 mol\%), $\mathrm{CH}_{2} \mathrm{Cl}_{2}(20 \mathrm{~mL}), 0{ }^{\circ} \mathrm{C}$ to RT, 1 h. ${ }^{a} 50 \mathrm{mmol}$ scale. ${ }^{\mathrm{b}} \mathbf{1 0 s}(21 \%) .{ }^{\mathrm{c}} \mathbf{1 0 t}(34 \%) .{ }^{\mathrm{d}} \mathbf{1 0 u}(27 \%) .{ }^{\mathrm{e}} \mathbf{1 0 z}(22 \%)$ (See Scheme 6 for structures).

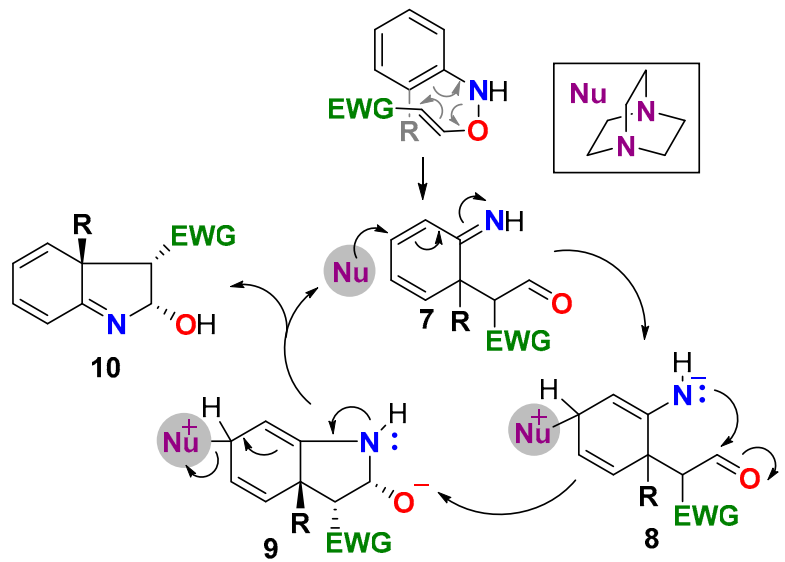

Scheme 6. Alternative [3,3]-sigmatropic rearrangement outcome of ortho-substituted $\mathrm{N}$-oxyenamines. 
As expected, substitution at the ortho-position posed a regioselectivity problem in the $[3,3]$-sigmatropic rearrangement, since the C-C bond formation at the most substituted ortho-position becomes a competitive pathway (Scheme 6). Indeed, in some cases, we were able to isolate the product $\mathbf{1 0}$ obtained from this pathway. The effect of this mechanistic divergence was mainly reflected in a diminished efficiency in the reactions of nitroarenes bearing a good leaving group at that position $(6 \mathbf{6 x}-\mathbf{z}$, $\mathbf{6 a a}-\mathbf{a b})$. In the case of simple alkyl substituents $(\mathbf{6 s}-\mathbf{u})$ or fused bicycles $(\mathbf{6 w})$, the manifold mostly afforded the expected C-7 substituted indole derivatives in moderate to good yields. The versatility of this methodology is highlighted when compared with Zhangs' protocol [56] (Scheme 1B), which is sensitive to steric impediments and shows a reduced tolerance to alkyl substitution at this position [70]. However, the easy access to ortho-substituted nitroarenes together with the simplicity of the 2-step reaction manifold compensate for these deficiencies and make this methodology a viable synthetic alternative to prepare C-7 substituted 3-EWG indoles.

The reaction manifold was well suited to scale up. As a proof of concept, we carried out the synthesis of methyl 6-acetylindole-3-carboxylate $(\mathbf{6 m})$ at $50 \mathrm{mmol}$ scale from 4-acetylnitrobenzene (3m) and methyl propiolate (5a). The scaled manifold afforded indole $6 \mathrm{~m}$ in an excellent $84 \%$ yield (9.08 g) (Scheme 5).

Finally, the anticancer activity of some 3-aroyl indoles [17,19] led us to evaluate our methodology as a novel and practical access to the chemical/therapeutic space of these derivatives. Among the huge number of possible 3-aroylindoles, we chose (6-methoxy-1H-indole-3-yl) (3,4,5-trimethoxyphenyl) methanone, the so-called BPRL0T075 (6ad, box in Scheme 5), an extremely potent cytotoxic compound with good pharmacological properties [21]. The manifold delivered it in a 30\% yield along with its C-4 isomer $(25 \%)$.

\section{Materials and Methods}

\subsection{General Information}

${ }^{1} \mathrm{H}-\mathrm{NMR}$ and ${ }^{13} \mathrm{C}-\mathrm{NMR}$ spectra (see supplementary materials) of DMSO-d6 or $\mathrm{CDCl}_{3}$ solutions were recorded either at 400 and $100 \mathrm{MHz}$ or at 500 and $125 \mathrm{MHz}$ (Bruker Ac 200 and AMX2-500 respectively). Mass spectra (low resolution) (EI/CI) were obtained with a Hewlett-Packard 5995 gas chromatograph/mass spectrometer. High-resolution mass spectra were recorded with a Micromass Autospec mass spectrometer. Analytical thin-layer chromatography plates used were E. Merck Brinkman UV-active silica gel (Kieselgel 60 F254) on aluminum. Flash column chromatography was carried out with E. Merck silica gel 60 (particle size less than $0.020 \mathrm{~mm}$ ) using appropriate mixtures of ethyl acetate and hexanes, or ethyl acetate and dichloromethane as eluents. All reactions were performed in oven-dried glassware. All materials were obtained from commercial suppliers and used as received. $N$-phenylhydroxylamine (4a) was prepared on a multigram scale following a standard experimental procedure [71].

\subsection{General Procedure for the Synthesis of Indoles 6 Directly from N-phenylhydroxylamine (4a). Preparation of} Methyl Indol-3-carboxylate (6a)

To a solution of $N$-phenylhydroxylamine $(4 \mathrm{a})(436 \mathrm{mg}, 4.0 \mathrm{mmol})$ in dichloromethane $(20 \mathrm{~mL})$ was added the activated alkyne $5(4.2 \mathrm{mmol})$ and the resulting solution was cooled to $0{ }^{\circ} \mathrm{C}$ using an ice bath. DABCO (22 mg, $0.2 \mathrm{mmol})$ was added all at once and the reaction mixture was stirred for $1 \mathrm{~h}$. The solvent was removed under reduced pressure, and the residue was purified by flash column chromatography (silica gel; n-hexane/ethyl acetate: 70/30 v/v) to give indole $6 a(565.2 \mathrm{mg}, 80 \%)$. Off-white solid: ${ }^{1} \mathrm{H}-\mathrm{NMR}\left(\mathrm{CDCl}_{3}, 400 \mathrm{MHz}\right): \delta=3.93(\mathrm{~s}, 3 \mathrm{H}), 7.25-7.28(\mathrm{~m}, 2 \mathrm{H}), 7.40-7.42(\mathrm{~m}, 1 \mathrm{H}), 7.91$ $(\mathrm{d}, 1 \mathrm{H}, J=3.0 \mathrm{~Hz}), 8.18-8.20(\mathrm{~m}, 1 \mathrm{H}), 8.82(\mathrm{bs}, 1 \mathrm{H}) .{ }^{13} \mathrm{C}-\mathrm{NMR}\left(\mathrm{CDCl}_{3}, 100 \mathrm{MHz}\right): \delta=51.1,108.7,111.5$, $121.5,122.0,123.2,125.6,131.1,136.1,165.8 \mathrm{ppm}$. The values are in accordance with the literature [72]. 
3.3. General Procedure for the Consecutive 2-Step Synthesis of Indoles $\mathbf{6}$ from Nitroarenes 3. Preparation of Methyl Indol-3-carboxylate (6a)

Under a nitrogen atmosphere, the nitrobenzene (3a) (4 mmol) was dissolved in dry tetrahydrofuran $(20 \mathrm{~mL})$ and $\mathrm{Rh} / \mathrm{C}(5 \%)(20 \mathrm{mg})$ was added to the solution. The resulting mixture was cooled to $0{ }^{\circ} \mathrm{C}$, hydrazine monohydrate $(250 \mathrm{mg}, 5 \mathrm{mmol})$ was then added dropwise and the reaction was stirred at $0{ }^{\circ} \mathrm{C}$ for $3 \mathrm{~h}$. The reaction mixture was filtered through a short pad of celite (aided with the use of dichloromethane) and the solvents evaporated under reduced pressure. The resulting crude product was dissolved in dichloromethane $(20 \mathrm{~mL})$ and methyl propiolate $(\mathbf{5 a})(344 \mathrm{mg}, 4.2 \mathrm{mmol})$ was added. The resulting solution was cooled to $0{ }^{\circ} \mathrm{C}$ using an ice bath and DABCO (22 $\left.\mathrm{mg}, 0.2 \mathrm{mmol}\right)$ was added at once. The reaction mixture was stirred for $1 \mathrm{~h}$ and the solvent was removed under reduced pressure. The crude residue was purified by flash column chromatography (silica gel; $n$-hexane/ethyl acetate: $70 / 30 v / v)$ to give indole $6 \mathbf{a}[72]$.

\subsection{Characterization Data for Indoles $\mathbf{6}$ and 3,3a-Dihydro-2H-Indoles $\mathbf{1 0}$}

Ethyl 1H-indole-3-carboxylate (6b) (330.2 mg, 87\%). Off-white solid: ${ }^{1} \mathrm{H}-\mathrm{NMR}$ (DMSO-d $6,500 \mathrm{MHz}$ ): $\delta=1.33(\mathrm{t}, 3 \mathrm{H}, J=7.1 \mathrm{~Hz}), 4.28(\mathrm{q}, 2 \mathrm{H}, J=7.1 \mathrm{~Hz}), 7.16-7.21(\mathrm{~m}, 2 \mathrm{H}), 7.47(\mathrm{dd}, 1 \mathrm{H}, J=6.5$ and $1.6 \mathrm{~Hz}), 8.00(\mathrm{dd}, 1 \mathrm{H}, J=6.8$ and $2.0 \mathrm{~Hz}), 8.06(\mathrm{~d}, 1 \mathrm{H}, J=2.3 \mathrm{~Hz}), 11.90(\mathrm{bs}, 1 \mathrm{H}) .{ }^{13} \mathrm{C}-\mathrm{NMR}\left(\mathrm{DMSO}_{6} \mathrm{~d}_{6}\right.$, $125 \mathrm{MHz}$ ): $\delta=14.5,59.0,106.6,112.3,120.5,121.2,122.3,125.6,132.4,136.4,164.4 \mathrm{ppm}$. The values are in accordance with the literature [73].

Octyl $1 \mathrm{H}$-indole-3-carboxylate (6c) $(217.5 \mathrm{mg}, 80 \%)$. Off-white solid: ${ }^{1} \mathrm{H}-\mathrm{NMR}\left(\mathrm{CDCl}_{3}, 400 \mathrm{MHz}\right)$ : $\delta=0.88(\mathrm{t}, 3 \mathrm{H}, J=6.8 \mathrm{~Hz}), 1.28-1.39(\mathrm{~m}, 8 \mathrm{H}), 1.43-1.47(\mathrm{~m}, 2 \mathrm{H}), 1.76-1.83(\mathrm{~m}, 2 \mathrm{H}), 4.34(\mathrm{t}, 2 \mathrm{H}, J=6.6 \mathrm{~Hz})$, 7.24-7.29 (m, 2H), 7.39-7.42 (m, 1Hz), $7.91(\mathrm{~d}, 1 \mathrm{H}, J=2.1 \mathrm{~Hz}), 8.16-8.20(\mathrm{~m}, 1 \mathrm{H}), 8.80(\mathrm{bs}, 1 \mathrm{H}) .{ }^{13} \mathrm{C}-\mathrm{NMR}$ $\left(\mathrm{CDCl}_{3}, 100 \mathrm{MHz}\right): \delta=14.0,22.6,26.2,29.0,29.2,29.3,31.8,64.1,109.2,111.5,121.6,122.0,123.1,125.8$, 131.0, 136.2, 165.5 ppm. HRMS (ESI $\left.{ }^{-}\right) \mathrm{m} / \mathrm{z}[\mathrm{M}-\mathrm{H}]^{-}$calculated for $\mathrm{C}_{17} \mathrm{H}_{22} \mathrm{NO}_{2}$ 272.1651, found 272.1650.

(1H-Indol-3-yl)(phenyl)methanone (6d) (351.4 mg, 83\%). Off-white solid: ${ }^{1} \mathrm{H}-\mathrm{NMR}$ (DMSO- $\mathrm{d}_{6}$, $400 \mathrm{MHz}): \delta=7.22-7.28(\mathrm{~m}, 2 \mathrm{H}), 7.52-7.62(\mathrm{~m}, 4 \mathrm{H}), 7.78-7.80(\mathrm{~m}, 2 \mathrm{H}), 7.93(\mathrm{~s}, 1 \mathrm{H}), 8.26(\mathrm{dd}, 1 \mathrm{H}, J=6.7$ and $1.7 \mathrm{~Hz}$ ), 12.10 (bs, $1 \mathrm{H}) .{ }^{13} \mathrm{C}-\mathrm{NMR}\left(\mathrm{DMSO}_{6}, 100 \mathrm{MHz}\right): \delta=112.2,115.0,121.4,121.9,123.1,126.2$, $128.3,128.4,131.0,136.7,140.5,190.0 \mathrm{ppm}$. The values are in accordance with the literature [74].

1-(1H-Indol-3-yl)hexan-1-one (6e) (144.5 mg, 67\%). Off-white solid: ${ }^{1} \mathrm{H}-\mathrm{NMR}\left(\mathrm{DMSO}-\mathrm{d}_{6}, 400 \mathrm{MHz}\right)$ : $\delta=0.87(\mathrm{t}, 3 \mathrm{H}, J=6.8 \mathrm{~Hz}), 1.29-1.34(\mathrm{~m}, 4 \mathrm{H}), 1.60-1.68(\mathrm{~m}, 2 \mathrm{H}), 2.82(\mathrm{t}, 2 \mathrm{H}, J=7.4 \mathrm{~Hz}), 7.14-7.22$ $(\mathrm{m}, 2 \mathrm{H}), 7.45-7.47(\mathrm{~m}, 1 \mathrm{H}), 8.20(\mathrm{dd}, 1 \mathrm{H}, J=6.9$ and $1.5 \mathrm{~Hz}), 8.30(\mathrm{~d}, 1 \mathrm{H}, J=3.0 \mathrm{~Hz}), 11.88(\mathrm{bs}, 1 \mathrm{H})$. ${ }^{13}$ C-NMR (DMSO-d 6,100 MHz): $\delta=13.9,22.0,24.6,31.1,38.7,112.0,116.4,121.4,121.5,122.6,125.4$, 133.6, 136.6, $195.5 \mathrm{ppm}$. The values are in accordance with the literature [75].

$\mathrm{N}$-cyclohexyl-1H-indole-3-carboxamide (6f) $(127.1 \mathrm{mg}, 52 \%)$. The reaction time was increased to overnight. Off-white solid: ${ }^{1} \mathrm{H}-\mathrm{NMR}\left(\mathrm{DMSO}-\mathrm{d}_{6}, 400 \mathrm{MHz}\right): \delta=1.12-1.36(\mathrm{~m}, 5 \mathrm{H}), 1.60-1.63(\mathrm{~m}, 1 \mathrm{H})$, 1.70-1.77 (m, 2H), 1.82-1.88 (m, 2H), 3.86-4.08 (m, 1H), 7.06-7.14 (m, 2H), $7.40(\mathrm{~d}, 1 \mathrm{H}, J=8.0 \mathrm{~Hz}), 7.60$ $(\mathrm{d}, 1 \mathrm{H}, J=6.1 \mathrm{~Hz}), 8.05(\mathrm{~s}, 1 \mathrm{H}), 8.14(\mathrm{~d}, 1 \mathrm{H}, J=7.8 \mathrm{~Hz}), 11.5(\mathrm{bs}, 1 \mathrm{H}) .{ }^{13} \mathrm{C}-\mathrm{NMR}\left(\mathrm{DMSO}-\mathrm{d}_{6}, 100 \mathrm{MHz}\right)$ : $\delta=25.0$ (2C), 25.4, 32.9 (2C), 47.4, 110.8, 111.6, 120.1, 121.1, 121.7, 126.3, 127.5, 136.1, 163.7 ppm. HRMS $\left(\mathrm{ESI}^{+}\right) \mathrm{m} / \mathrm{z}[\mathrm{M}+\mathrm{Na}]^{+}$calculated for $\mathrm{C}_{15} \mathrm{H}_{18} \mathrm{~N}_{2} \mathrm{ONa} 265.1317$, found 265.1314. The product had been previously described in the literature but in $\mathrm{CDCl}_{3}$ [76].

N-Methyl-N-phenyl-1H-indole-3-carboxamide (6g) (290.4 mg, 58\%). The reaction time was increased to overnight. Off-white solid: ${ }^{1} \mathrm{H}-\mathrm{NMR}\left(\mathrm{DMSO}_{6} \mathrm{~d}_{6}, 500 \mathrm{MHz}\right): \delta=3.36(\mathrm{~s}, 3 \mathrm{H}), 6.36(\mathrm{~d}, 1 \mathrm{H}, \mathrm{J}=3.0 \mathrm{~Hz})$, $7.07(\mathrm{dd}, 1 \mathrm{H}, J=7.4$ and $1.2 \mathrm{~Hz}), 7.11(\mathrm{dd}, 1 \mathrm{H}, J=7.5$ and $1.5 \mathrm{~Hz}), 7.28-7.32(\mathrm{~m}, 4 \mathrm{H}), 7.37-7.40(\mathrm{~m}, 2 \mathrm{H})$, $8.04(\mathrm{~d}, 1 \mathrm{H}, \mathrm{J}=8.0 \mathrm{~Hz}), 11.28$ (bs, $1 \mathrm{H}) .{ }^{13} \mathrm{C}-\mathrm{NMR}\left(\mathrm{DMSO}-\mathrm{d}_{6}, 125 \mathrm{MHz}\right): \delta=37.9,109.6,111.5,120.3$, $121.3,121.9,126.8,127.2,127.5(2 \mathrm{C}), 128.9,129.4(2 \mathrm{C}), 135.1,145.5,165.1 \mathrm{ppm}$. The values are in accordance with the literature [77].

Methyl 5-methyl-1H-indole-3-carboxylate (6h) (533.5 mg, 77\%). Off-white solid: ${ }^{1} \mathrm{H}-\mathrm{NMR}\left(\mathrm{CDCl}_{3}\right.$, $400 \mathrm{MHz}): \delta=2.48(\mathrm{~s}, 3 \mathrm{H}), 3.92(\mathrm{~s}, 3 \mathrm{H}), 7.08(\mathrm{dd}, 1 \mathrm{H}, J=8.3$ and $1.2 \mathrm{~Hz}), 7.29(\mathrm{~d}, 1 \mathrm{H}, J=8.3 \mathrm{~Hz}), 7.86$ $(\mathrm{d}, 1 \mathrm{H}, J=3.0 \mathrm{~Hz}), 7.98(\mathrm{~d}, 1 \mathrm{H}, J=1.2 \mathrm{~Hz}), 8.72(\mathrm{bs}, 1 \mathrm{H}) .{ }^{13} \mathrm{C}-\mathrm{NMR}\left(\mathrm{CDCl}_{3}, 100 \mathrm{MHz}\right): \delta=21.5,51.0$, 
$108.2,111.1,121.1,124.8,126.0,131.1,131.6,134.4,165.8 \mathrm{ppm}$. The values are in accordance with the literature [78].

Methyl 5-chloro-1H-indole-3-carboxylate (6i) $\left(309.3 \mathrm{mg}, 78 \%\right.$ ). Off-white solid: ${ }^{1} \mathrm{H}-\mathrm{NMR}$ (DMSO- $\mathrm{d}_{6}$, $400 \mathrm{MHz}): \delta=4.36(\mathrm{~s}, 3 \mathrm{H}), 7.74(\mathrm{~d}, 1 \mathrm{H}, J=8.6 \mathrm{~Hz}), 8.05(\mathrm{~d}, 1 \mathrm{H}, J=8.6 \mathrm{~Hz}), 8.55(\mathrm{~s}, 1 \mathrm{H}), 8.69(\mathrm{~s}, 1 \mathrm{H})$, 12.65 (s, 1H). ${ }^{13} \mathrm{C}-\mathrm{NMR}$ (DMSO-d $6,100 \mathrm{MHz}$ ): $\delta=51.6,107.2,114.8,120.6,123.4,127.1,127.8,134.7$, $135.9,165.4$. The values are in accordance with the literature [73].

Methyl 5-bromo-1H-indole-3-carboxylate $(\mathbf{6 j})(820.0 \mathrm{mg}, 81 \%)$. Off-white solid: ${ }^{1} \mathrm{H}-\mathrm{NMR}\left(\mathrm{CDCl}_{3}\right.$, $400 \mathrm{MHz}): \delta=3.90(\mathrm{~s}, 3 \mathrm{H}), 7.28(\mathrm{~d}, 1 \mathrm{H}, J=8.6 \mathrm{~Hz}), 7.36(\mathrm{dd}, 1 \mathrm{H}, J=8.6$ and $1.8 \mathrm{~Hz}), 7.91(\mathrm{~d}, 1 \mathrm{H}$, $J=2.9 \mathrm{~Hz}), 8.32(\mathrm{~d}, 1 \mathrm{H}, J=1.8 \mathrm{~Hz}), 8.61(\mathrm{bs}, 1 \mathrm{H}) .{ }^{13} \mathrm{C}-\mathrm{NMR}\left(\mathrm{CDCl}_{3}, 100 \mathrm{MHz}\right): \delta=51.2,108.8,112.9$, $115.7,124.3,126.3,127.4,131.7,134.7,165.1 \mathrm{ppm}$. The values are in accordance with the literature [79].

Methyl 5-iodo-1H-indole-3-carboxylate (6k) $(542.9 \mathrm{mg}, 90 \%)$. Off-white solid: ${ }^{1} \mathrm{H}-\mathrm{NMR}$ (DMSO-d $\mathrm{d}_{6}$, $400 \mathrm{MHz}): \delta=3.81(\mathrm{~s}, 3 \mathrm{H}), 7.34(\mathrm{~d}, 1 \mathrm{H}, J=8.5 \mathrm{~Hz}), 7.48(\mathrm{dd}, 1 \mathrm{H}, J=8.5$ and $1.5 \mathrm{~Hz}), 8.09(\mathrm{~d}, 1 \mathrm{H}$, $J=2.9 \mathrm{~Hz}), 8.33(\mathrm{~d}, 1 \mathrm{H}, J=1.3 \mathrm{~Hz}), 12.09$ (bs, $1 \mathrm{H}) .{ }^{13} \mathrm{C}-\mathrm{NMR}\left(\mathrm{DMSO}_{-} \mathrm{d}_{6}, 100 \mathrm{MHz}\right): \delta=50.8,85.7,105.7$, $114.8,128.1,128.7,130.4,133.2,135.5,164.4$. ppm. The values are in accordance with the literature [80].

Methyl 5-methoxy-1H-indole-3-carboxylate (61). (214.2 mg, 52\%). Off-white solid: ${ }^{1} \mathrm{H}-\mathrm{NMR}\left(\mathrm{CDCl}_{3}\right.$, $400 \mathrm{MHz}): \delta=3.87(\mathrm{~s}, 3 \mathrm{H}), 3.91(\mathrm{~s}, 3 \mathrm{H}), 6.90(\mathrm{dd}, 1 \mathrm{H}, J=8.8$ and $2.5 \mathrm{~Hz}), 7.28(\mathrm{~d}, 1 \mathrm{H}, J=8.8 \mathrm{~Hz}), 7.66$ $(\mathrm{d}, 1 \mathrm{H}, J=2.5 \mathrm{~Hz}), 7.85(\mathrm{~d}, 1 \mathrm{H}, J=3.0 \mathrm{~Hz}), 8.74(\mathrm{bs}, 1 \mathrm{H}) .{ }^{13} \mathrm{C}-\mathrm{NMR}\left(\mathrm{CDCl}_{3}, 100 \mathrm{MHz}\right): \delta=51.0,55.8$, $102.8,108.3,112.3,113.7,126.7,131.0,131.2,155.9,165.8 \mathrm{ppm}$. The values are in accordance with the literature [79].

Methyl 5-acetyl-1H-indole-3-carboxylate (6m). (9.083 g, 84\%). Off-white solid: ${ }^{1} \mathrm{H}-\mathrm{NMR}\left(\mathrm{DMSO}-\mathrm{d}_{6}\right.$, $400 \mathrm{MHz}): \delta=2.63(\mathrm{~s}, 3 \mathrm{H}), 3.84(\mathrm{~s}, 3 \mathrm{H}), 7.56(\mathrm{~d}, 1 \mathrm{H}, J=8.6 \mathrm{~Hz}), 7.84(\mathrm{dd}, 1 \mathrm{H}, J=8.6$ and $1.5 \mathrm{~Hz}), 8.22$ $(\mathrm{d}, 1 \mathrm{H}, J=3.0 \mathrm{~Hz}), 8.65(\mathrm{~d}, 1 \mathrm{H}, J=1.5 \mathrm{~Hz}), 12.25$ (bs, $1 \mathrm{H}) .{ }^{13} \mathrm{C}-\mathrm{NMR}\left(\mathrm{DMSO}-\mathrm{d}_{6}, 100 \mathrm{MHz}\right): \delta=26.7$, 50.8, 107.7, 112.3, 122.0, 122.5, 125.2, 130.8, 134.2, 138.9, 164.4, 197.4 ppm. HRMS $\left(\mathrm{ESI}^{+}\right) \mathrm{m} / \mathrm{z}\left[\mathrm{M}+\mathrm{Na}^{+}\right.$ calculated for $\mathrm{C}_{12} \mathrm{H}_{11} \mathrm{NO}_{3} \mathrm{Na} 240.0637$, found 240.0634 .

Methyl 4-methyl-1H-indole-3-carboxylate (6n; C-4 isomer) (348.7 mg, 46\%). Off-white solid: ${ }^{1} \mathrm{H}-\mathrm{NMR}$ $\left(\mathrm{CDCl}_{3}, 400 \mathrm{MHz}\right): \delta=2.87(\mathrm{~s}, 3 \mathrm{H}), 3.87(\mathrm{~s}, 3 \mathrm{H}), 7.03(\mathrm{~d}, 1 \mathrm{H}, J=7.1 \mathrm{~Hz}), 7.15(\mathrm{t}, 1 \mathrm{H}, J=7.6 \mathrm{~Hz}), 7.22$ $(\mathrm{d}, 1 \mathrm{H}, J=8.0 \mathrm{~Hz}), 7.89(\mathrm{~d}, 1 \mathrm{H}, J=3.0 \mathrm{~Hz}), 8.76(\mathrm{bs}, 1 \mathrm{H}) .{ }^{13} \mathrm{C}-\mathrm{NMR}\left(\mathrm{CDCl}_{3}, 100 \mathrm{MHz}\right): \delta=22.3,51.1$, $109.2,109.5,123.4,124.0,124.4,132.1,132.4,136.9,165.4 \mathrm{ppm}$. The values are in accordance with the literature [81].

Methyl 6-methyl-1H-indole-3-carboxylate (6n; C-6 isomer) (257.7 mg, 34\%). Off-white solid: ${ }^{1} \mathrm{H}-\mathrm{NMR}$ $\left(\mathrm{CDCl}_{3}, 400 \mathrm{MHz}\right): \delta=2.45(\mathrm{~s}, 3 \mathrm{H}), 3.91(\mathrm{~s}, 3 \mathrm{H}), 7.10(\mathrm{~d}, 1 \mathrm{H}, J=8.0 \mathrm{~Hz}), 7.18(\mathrm{~s}, 1 \mathrm{H}), 7.83(\mathrm{~d}, 1 \mathrm{H}$, $J=3.0 \mathrm{~Hz}), 8.04(\mathrm{~d}, 1 \mathrm{H}, J=8.0 \mathrm{~Hz}), 8.70(\mathrm{bs}, 1 \mathrm{H}) .{ }^{13} \mathrm{C}-\mathrm{NMR}\left(\mathrm{CDCl}_{3}, 100 \mathrm{MHz}\right): \delta=21.6,51.0,108.5,111.4$, 121.0, 123.6, 123.8, 130.5, 133.1, 136.5, $165.8 \mathrm{ppm}$. The values are in accordance with the literature [81].

Methyl 4-chloro-1H-indole-3-carboxylate (6o; C-4 isomer) (186.0 mg, 44\%). Pale yellow solid: ${ }^{1} \mathrm{H}-\mathrm{NMR}\left(\right.$ DMSO-d $\left._{6}, 400 \mathrm{MHz}\right) \delta=3.76(\mathrm{~s}, 3 \mathrm{H}), 7.13-7.26(\mathrm{~m}, 2 \mathrm{H}), 7.46(\mathrm{dd}, 1 \mathrm{H}, J=6.7$ and $2.3 \mathrm{~Hz})$, $8.13(\mathrm{~d}, 1 \mathrm{H}, J=2.6 \mathrm{~Hz}), 12.18(\mathrm{~s}, 1 \mathrm{H}) .{ }^{13} \mathrm{C}-\mathrm{NMR}\left(\mathrm{DMSO}_{-} \mathrm{d}_{6}, 100 \mathrm{MHz}\right) \delta=51.8,107.8,112.4,123.3,123.6$, 124.1, 125.8, 135.0, 139.3, 164.4. HRMS (ESI $\left.{ }^{-}\right) \mathrm{m} / \mathrm{z}[\mathrm{M}-\mathrm{H}]^{-}$calculated for $\mathrm{C}_{10} \mathrm{H}_{7} \mathrm{NO}_{2} \mathrm{Cl} 208.0165$, found 208.0163.

Methyl 6-chloro-1H-indole-3-carboxylate (6o; C-6 isomer) (175.6 mg, 42\%). Pale yellow solid: ${ }^{1} \mathrm{H}-\mathrm{NMR}\left(\mathrm{CDCl}_{3}, 400 \mathrm{MHz}\right) \delta=3.94(\mathrm{~s}, 3 \mathrm{H}), 7.26(\mathrm{~d}, 1 \mathrm{H}, J=9.4 \mathrm{~Hz}), 7.43(\mathrm{~s}, 1 \mathrm{H}), 7.92(\mathrm{~s}, 1 \mathrm{H}), 8.11$ $(\mathrm{d}, 1 \mathrm{H}, J=8.6 \mathrm{~Hz}), 8.60(\mathrm{~s}, 1 \mathrm{H}) .{ }^{13} \mathrm{C}-\mathrm{NMR}$ (DMSO- $\left.d_{6}, 100 \mathrm{MHz}\right) \delta=52.7,108.3,113.8,123.4,123.5,125.9$, 129.0, 135.1, 138.4, 166.7. The values are in accordance with the literature [62].

Methyl 4-bromo-1H-indole-3-carboxylate (6p; C-4 isomer) (258.8 mg, 51\%). Pale yellow solid: ${ }^{1} \mathrm{H}-\mathrm{NMR}\left(\mathrm{DMSO}_{\mathrm{d}}, 400 \mathrm{MHz}\right) \delta=3.77(\mathrm{~s}, 3 \mathrm{H}), 7.11(\mathrm{t}, 1 \mathrm{H}, J=7.9 \mathrm{~Hz}), 7.39(\mathrm{~d}, 1 \mathrm{H}, J=7.5 \mathrm{~Hz}), 7.51$ $(\mathrm{d}, 1 \mathrm{H}, J=7.8 \mathrm{~Hz}), 8.12(\mathrm{~s}, 1 \mathrm{H}), 12.16(\mathrm{~s}, 1 \mathrm{H}) .{ }^{13} \mathrm{C}-\mathrm{NMR}\left(\mathrm{DMSO}_{\mathrm{d}}, 100 \mathrm{MHz}\right) \delta=51.7,108.4,112.9,113.7$, 124.4, 125.0, 127.1, 134.7, 139.0, 164.5. HRMS $\left(\mathrm{ESI}^{+}\right) \mathrm{m} / \mathrm{z}[\mathrm{M}+\mathrm{Na}]^{+}$calculated for $\mathrm{C}_{10} \mathrm{H}_{8} \mathrm{NO}_{2} \mathrm{BrNa}$ 275.9636 , found 275.9642 .

Methyl 6-bromo-1H-indole-3-carboxylate (6p; C-6 isomer) (200.1 mg, 39\%). Pale yellow solid: ${ }^{1} \mathrm{H}-\mathrm{NMR}\left(\mathrm{DMSO}_{6} \mathrm{~d}_{6}, 400 \mathrm{MHz}\right) \delta=3.80(\mathrm{~s}, 3 \mathrm{H}), 7.32(\mathrm{dd}, 1 \mathrm{H}, J=8.5$ and $1.8 \mathrm{~Hz}), 7.67(\mathrm{~s}, 1 \mathrm{H}), 7.92$ $(\mathrm{d}, 1 \mathrm{H}, J=8.5 \mathrm{~Hz}), 8.11(\mathrm{~d}, 1 \mathrm{H}, J=2.8 \mathrm{~Hz}), 12.03(\mathrm{~s}, 1 \mathrm{H}) .{ }^{13} \mathrm{C}-\mathrm{NMR}\left(\right.$ DMSO-d $\left._{6}, 100 \mathrm{MHz}\right) \delta=51.7,107.5$, 
115.9, 115.9, 123, 125.1, 125.6, 134.2, 138.1, 165.3. HRMS (ESI') $\mathrm{m} / \mathrm{z}[\mathrm{M}-\mathrm{H}]^{-}$calculated for $\mathrm{C}_{10} \mathrm{H}_{7} \mathrm{NO}_{2} \mathrm{Br}$ 251.9660, found 251.9657.

Methyl 4-acetyl-1H-indole-3-carboxylate (6q; C-4 isomer) (539.8 mg, 62\%). Off-white solid: ${ }^{1} \mathrm{H}-\mathrm{NMR}$ (DMSO- $\left.d_{6}, 400 \mathrm{MHz}\right): \delta=2.45(\mathrm{~s}, 3 \mathrm{H}), 3.72(\mathrm{~s}, 3 \mathrm{H}), 7.18(\mathrm{~d}, 1 \mathrm{H}, J=7.2 \mathrm{~Hz}), 7.26(\mathrm{t}, 1 \mathrm{H}, J=7.5 \mathrm{~Hz}), 7.60$ $(\mathrm{d}, 1 \mathrm{H}, J=7.9 \mathrm{~Hz}), 8.14(\mathrm{~s}, 1 \mathrm{H}), 12.17(\mathrm{bs}, 1 \mathrm{H}) .{ }^{13} \mathrm{C}-\mathrm{NMR}\left(\mathrm{CDCl}_{3}, 100 \mathrm{MHz}\right): \delta=31.1,51.0,108.2,114.3$, 119.8, 120.7, 122.5, 132.6, 135.3, 137.0, 165.2, $205.2 \mathrm{ppm}$. HRMS $\left(\mathrm{ESI}^{+}\right) \mathrm{m} / \mathrm{z}[\mathrm{M}+\mathrm{Na}]^{+}$calculated for $\mathrm{C}_{12} \mathrm{H}_{11} \mathrm{NO}_{3} \mathrm{Na} 240.0637$, found 240.0634.

Methyl 6-acetyl-1H-indole-3-carboxylate (6q; C-6 isomer) (237.7 mg, 27\%). Off-white solid: ${ }^{1} \mathrm{H}-\mathrm{NMR}$ (DMSO-d $\left.\mathrm{d}_{6}, 400 \mathrm{MHz}\right): \delta=2.66(\mathrm{~s}, 3 \mathrm{H}), 3.86(\mathrm{~s}, 3 \mathrm{H}), 7.84(\mathrm{dd}, 1 \mathrm{H}, J=8.4$ and $1.5 \mathrm{~Hz}), 8.10(\mathrm{~d}, 1 \mathrm{H}$, $J=8.4 \mathrm{~Hz}), 8.15(\mathrm{~d}, 1 \mathrm{H}, J=1.5 \mathrm{~Hz}), 8.33(\mathrm{~d}, 1 \mathrm{H}, J=3.0 \mathrm{~Hz}), 12.39(\mathrm{bs}, 1 \mathrm{H}) .{ }^{13} \mathrm{C}-\mathrm{NMR}$ (DMSO- $d_{6}$, $100 \mathrm{MHz}): \delta=26.7,50.8,106.7,113.4,120.1,121.1,129.9,131.5,135.7,135.9,164.4,197.3$ ppm. HRMS $\left(\mathrm{ESI}^{+}\right) \mathrm{m} / \mathrm{z}[\mathrm{M}+\mathrm{Na}]^{+}$calculated for $\mathrm{C}_{12} \mathrm{H}_{11} \mathrm{NO}_{3} \mathrm{Na} 240.0637$, found 240.0640 .

Methyl 4-methoxy- $1 \mathrm{H}$-indole-3-carboxylate (6r; C-4 isomer) $(111.8 \mathrm{mg}, 27 \%)$. Off-white solid: ${ }^{1} \mathrm{H}-\mathrm{NMR}\left(\mathrm{CDCl}_{3}, 400 \mathrm{MHz}\right): \delta=3.86(\mathrm{~s}, 3 \mathrm{H}), 3.93(\mathrm{~s}, 3 \mathrm{H}), 6.66(\mathrm{~d}, 1 \mathrm{H}, J=7.8 \mathrm{~Hz}), 7.00(\mathrm{~d}, 1 \mathrm{H}, J=8.0 \mathrm{~Hz})$, $7.16(\mathrm{t}, 1 \mathrm{H}, J=7.0 \mathrm{~Hz}), 7.81(\mathrm{~d}, 1 \mathrm{H}, J=2.8 \mathrm{~Hz}), 9.11(\mathrm{bs}, 1 \mathrm{H}) .{ }^{13} \mathrm{C}-\mathrm{NMR}\left(\mathrm{CDCl}_{3}, 100 \mathrm{MHz}\right): \delta=51.3,55.9$, $102.9,105.0,108.6,115.4,124.2,131.3,138.4,154.2,165.0 \mathrm{ppm}$. HRMS $\left(\mathrm{ESI}^{+}\right) \mathrm{m} / \mathrm{z}[\mathrm{M}+\mathrm{Na}]^{+}$calculated for $\mathrm{C}_{11} \mathrm{H}_{11} \mathrm{NO}_{3} \mathrm{Na} 228.0637$, found 228.0634 .

Methyl 6-methoxy-1H-indole-3-carboxylate (6r; C-6 isomer) (189.5 mg, 46\%). Off-white solid: ${ }^{1} \mathrm{H}-\mathrm{NMR}$ (DMSO- $\left.\mathrm{d}_{6}, 400 \mathrm{MHz}\right): \delta=3.78(\mathrm{~s}, 6 \mathrm{H}), 6.83(\mathrm{dd}, 1 \mathrm{H}, J=8.6$ and $2.2 \mathrm{~Hz}), 6.96(\mathrm{~d}, 1 \mathrm{H}, J=2.2 \mathrm{~Hz}), 7.84$ $(\mathrm{d}, 1 \mathrm{H}, J=8.6 \mathrm{~Hz}), 7.94(\mathrm{~d}, 1 \mathrm{H}, J=3.0 \mathrm{~Hz}), 11.70(\mathrm{bs}, 1 \mathrm{H}) .{ }^{13} \mathrm{C}-\mathrm{NMR}$ (DMSO- $\left.d_{6}, 100 \mathrm{MHz}\right): \delta=50.5$, $55.2,95.2,106.3,111.3,119.7,121.0,131.2,137.1,156.1,164.7 \mathrm{ppm}$. The values are in accordance with the literature [82].

Methyl 7-methyl-1H-indole-3-carboxylate (6s) (245.9.5 mg, 65\%). Off-white solid: ${ }^{1} \mathrm{H}-\mathrm{NMR}\left(\mathrm{CDCl}_{3}\right.$, $400 \mathrm{MHz}): \delta=2.50(\mathrm{~s}, 3 \mathrm{H}), 3.92(\mathrm{~s}, 3 \mathrm{H}), 7.06(\mathrm{~d}, 1 \mathrm{H}, J=7.1 \mathrm{~Hz}), 7.19(\mathrm{t}, 1 \mathrm{H}, J=7.6 \mathrm{~Hz}), 7.90(\mathrm{~d}, 1 \mathrm{H}$, $J=2.8 \mathrm{~Hz}), 8.02(\mathrm{~d}, 1 \mathrm{H}, J=8.0 \mathrm{~Hz}), 8.71(\mathrm{bs}, 1 \mathrm{H}) .{ }^{13} \mathrm{C}-\mathrm{NMR}\left(\mathrm{CDCl}_{3}, 100 \mathrm{MHz}\right): \delta=16.5,51.1,109.2,119.2$, $120.7,122.2,123.8,125.4,130.7,135.7,165.8 \mathrm{ppm}$. The values are in accordance with the literature [83].

Methyl 6,7-dimethyl-1H-indole-3-carboxylate (6t) $(235.0 \mathrm{mg}, 58 \%)$. Off-white solid: ${ }^{1} \mathrm{H}-\mathrm{NMR}$ (DMSO-d $6,400 \mathrm{MHz}): \delta=2.32(\mathrm{~s}, 3 \mathrm{H}), 2.39(\mathrm{~s}, 3 \mathrm{H}), 3.79(\mathrm{~s}, 3 \mathrm{H}), 6.99(\mathrm{~d}, 1 \mathrm{H}, J=8.0 \mathrm{~Hz}), 7.71(\mathrm{~d}, 1 \mathrm{H}$, $J=8.0 \mathrm{~Hz}), 7.99(\mathrm{~d}, 1 \mathrm{H}, J=3.0 \mathrm{~Hz}), 11.78(\mathrm{bs}, 1 \mathrm{H}) .{ }^{13} \mathrm{C}-\mathrm{NMR}\left(\mathrm{DMSO}_{\mathrm{d}}, 100 \mathrm{MHz}\right): \delta=13.1,18.9,50.5$, 106.6, 117.4, 119.4, 123.8, 123.9, 129.5, 131.6, 136.5, 164.8 ppm. HRMS $\left(\mathrm{ESI}^{+}\right) \mathrm{m} / \mathrm{z}[\mathrm{M}+\mathrm{Na}]^{+}$calculated for $\mathrm{C}_{12} \mathrm{H}_{13} \mathrm{NO}_{2} \mathrm{Na} 226.0844$, found 226.0845 .

Methyl 6-chloro-7-methyl-1H-indole-3-carboxylate (6u) (299.8 mg, 67\%). Off-white solid: ${ }^{1} \mathrm{H}-\mathrm{NMR}$ $\left(\mathrm{CDCl}_{3}, 400 \mathrm{MHz}\right): \delta=2.52(\mathrm{~s}, 3 \mathrm{H}), 3.91(\mathrm{~s}, 3 \mathrm{H}), 7.26(\mathrm{~d}, 1 \mathrm{H}, J=8.6 \mathrm{~Hz}), 7.90(\mathrm{~d}, 1 \mathrm{H}, J=2.9 \mathrm{~Hz}), 7.93$ $(\mathrm{d}, 1 \mathrm{H}, J=8.6 \mathrm{~Hz}), 8.56$ (bs, $1 \mathrm{H}) .{ }^{13} \mathrm{C}-\mathrm{NMR}\left(\mathrm{CDCl}_{3}, 100 \mathrm{MHz}\right): \delta=13.8,51.2,109.6,118.6,119.8,123.5$, 124.1, 128.9, 131.0, 136.2, 165.3 ppm. HRMS (ESI $\left.{ }^{-}\right) \mathrm{m} / \mathrm{z}[\mathrm{M}-\mathrm{H}]^{-}$calculated for $\mathrm{C}_{11} \mathrm{H}_{9} \mathrm{ClNO}_{2} 222.0322$, found 222.0314 .

Methyl 4,6-dimethyl-1H-indole-3-carboxylate (6v) $(607.7 \mathrm{mg}, 75 \%)$. Off-white solid: ${ }^{1} \mathrm{H}-\mathrm{NMR}$ (DMSO-d $\left.\mathrm{d}_{6}, 400 \mathrm{MHz}\right): \delta=2.33(\mathrm{~s}, 3 \mathrm{H}), 2.70(\mathrm{~s}, 3 \mathrm{H}), 3.73(\mathrm{~s}, 3 \mathrm{H}), 6.74(\mathrm{~s}, 1 \mathrm{H}), 7.07(\mathrm{~s}, 1 \mathrm{H}), 7.96(\mathrm{~d}, 1 \mathrm{H}$, $J=3.0 \mathrm{~Hz}), 11.71(\mathrm{bs}, 1 \mathrm{H}) .{ }^{13} \mathrm{C}-\mathrm{NMR}\left(\right.$ DMSO- $\left._{6}, 100 \mathrm{MHz}\right): \delta=20.9,21.9,50.6,107.2,109.7,122.1,124.9$, $130.3,131.6,132.7,137.6,164.6 \mathrm{ppm}$. HRMS (ESI') $\mathrm{m} / \mathrm{z}[\mathrm{M}-\mathrm{H}]^{-}$calculated for $\mathrm{C}_{12} \mathrm{H}_{12} \mathrm{NO}_{2} 202.0868$, found 202.0872 .

Methyl 1,6,7,8-tetrahydrocyclopenta[g]indole-3-carboxylate (6w) (305.6 mg, 71\%). Off-white solid: ${ }^{1} \mathrm{H}-\mathrm{NMR}\left(\mathrm{DMSO}_{6}, 400 \mathrm{MHz}\right): \delta=2.08-2.15(\mathrm{~m}, 2 \mathrm{H}), 2.95(\mathrm{t}, 2 \mathrm{H}, J=7.3 \mathrm{~Hz}), 3.04(\mathrm{t}, 2 \mathrm{H}, J=7.3 \mathrm{~Hz})$, $3.79(\mathrm{~s}, 3 \mathrm{H}), 7.08(\mathrm{~d}, 1 \mathrm{H}, J=8.0 \mathrm{~Hz}), 7.79(\mathrm{~d}, 1 \mathrm{H}, J=8.0 \mathrm{~Hz}), 7.99(\mathrm{~d}, 1 \mathrm{H}, J=3.0 \mathrm{~Hz}), 11.88(\mathrm{bs}, 1 \mathrm{H})$. ${ }^{13} \mathrm{C}-\mathrm{NMR}$ (DMSO-d ${ }_{6}, 100 \mathrm{MHz}$ ): $\delta=25.0,29.9,32.6,50.6,106.9,118.1,118.5,124.4,126.5,131.6,133.5$, 138.3, 164.9 ppm. HRMS (ESI $\left.{ }^{+}\right) \mathrm{m} / \mathrm{z}[\mathrm{M}+\mathrm{Na}]^{+}$calculated for $\mathrm{C}_{13} \mathrm{H}_{13} \mathrm{NO}_{2} \mathrm{Na} 238.0844$, found 238.0848.

Methyl 7-chloro-1H-indole-3-carboxylate (6x) $(175.7 \mathrm{mg}, 21 \%)$. Pale yellow solid: ${ }^{1} \mathrm{H}-\mathrm{NMR}$ (DMSO- $\mathrm{d}_{6}$, $400 \mathrm{MHz}): \delta=3.82(\mathrm{~s}, 3 \mathrm{H}), 7.18(\mathrm{t}, 1 \mathrm{H}, J=7.8 \mathrm{~Hz}), 7.29(\mathrm{dd}, 1 \mathrm{H}, J=7.7$ and $1.1 \mathrm{~Hz}), 7.98(\mathrm{~d}, 1 \mathrm{H}$, $J=7.9 \mathrm{~Hz}), 8.11(\mathrm{~d}, 1 \mathrm{H}, J=3.1 \mathrm{~Hz}), 12.36(\mathrm{~s}, 1 \mathrm{H}) .{ }^{13} \mathrm{C}-\mathrm{NMR}\left(\mathrm{DMSO}^{-} \mathrm{d}_{6}, 100 \mathrm{MHz}\right) \delta=51.7,108.6,117.6$, 
120.4, 122.9, 123.3, 128.4, 134.1, 134.2, 165.3. HRMS (ESI-) $\mathrm{m} / \mathrm{z}[\mathrm{M}-\mathrm{H}]^{-}$calculated for $\mathrm{C}_{10} \mathrm{H}_{7} \mathrm{NO}_{2} \mathrm{Cl}$ 208.0165, found 208.0158.

Methyl 7-fluoro-1H-indole-3-carboxylate (6y) $(285.6 \mathrm{mg}, 37 \%)$. Off-white solid: ${ }^{1} \mathrm{H}-\mathrm{NMR}\left(\mathrm{DMSO}-\mathrm{d}_{6}\right.$, $400 \mathrm{MHz}): \delta=3.81(\mathrm{~s}, 3 \mathrm{H}), 7.05(\mathrm{dd}, 1 \mathrm{H}, J=11.5$ and $8.0 \mathrm{~Hz}), 7.13-7.18(\mathrm{~m}, 1 \mathrm{H}), 7.81(\mathrm{~d}, 1 \mathrm{H}, J=8.0 \mathrm{~Hz})$, $8.12(\mathrm{~s}, 1 \mathrm{H}), 12.49$ (bs, $1 \mathrm{H}) .{ }^{13} \mathrm{C}-\mathrm{NMR}$ (DMSO-d $\left.{ }_{6}, 100 \mathrm{MHz}\right): \delta=50.8,107.3(\mathrm{~d}, J=16 \mathrm{~Hz}), 107.4,116.5$ $(\mathrm{d}, J=3.5 \mathrm{~Hz}), 121.8(\mathrm{~d}, J=6.3 \mathrm{~Hz}), 124.2(\mathrm{~d}, J=13.5 \mathrm{~Hz}), 129.3(\mathrm{~d}, J=5.3 \mathrm{~Hz}), 133.1,149.2(\mathrm{~d}, J=244 \mathrm{~Hz})$, 164.4 ppm. HRMS (ESI') $\mathrm{m} / \mathrm{z}[\mathrm{M}-\mathrm{H}]^{-}$calculated for $\mathrm{C}_{10} \mathrm{H}_{7} \mathrm{FNO}_{2}$ 192.0461, found 192.0457.

Methyl 7-methoxy-1H-indole-3-carboxylate (6z) $(90.4 \mathrm{mg}, 11 \%)$. Off-white solid: ${ }^{1} \mathrm{H}-\mathrm{NMR}\left(\mathrm{CDCl}_{3}\right.$, $500 \mathrm{MHz}): \delta=3.91(\mathrm{~s}, 3 \mathrm{H}), 3.94(\mathrm{~s}, 3 \mathrm{H}), 6.70(\mathrm{~d}, 1 \mathrm{H}, J=7.8 \mathrm{~Hz}), 7.17(\mathrm{t}, 1 \mathrm{H}, J=8.0 \mathrm{~Hz}), 7.75(\mathrm{~d}, 1 \mathrm{H}$, $J=8.0 \mathrm{~Hz}), 7.87(\mathrm{~d}, 1 \mathrm{H}, J=3.0 \mathrm{~Hz}), 8.90(\mathrm{bs}, 1 \mathrm{H}) .{ }^{13} \mathrm{C}-\mathrm{NMR}\left(\mathrm{CDCl}_{3}, 125 \mathrm{MHz}\right): \delta=51.0,55.4,102.9,109.2$, $113.9,122.5,126.7,127.1,130.3,146.1,165.7 \mathrm{ppm}$. The values are in accordance with the literature [84].

Methyl 7-chloro-4-fluoro-1H-indole-3-carboxylate (6aa) (245.2 mg, 27\%). Off-white solid: ${ }^{1} \mathrm{H}-\mathrm{NMR}$ $\left(\mathrm{CDCl}_{3}, 400 \mathrm{MHz}\right): \delta=3.90(\mathrm{~s}, 3 \mathrm{H}), 6.87(\mathrm{dd}, 1 \mathrm{H}, J=10.5$ and $8.5 \mathrm{~Hz}), 7.16(\mathrm{dd}, 1 \mathrm{H}, J=8.5$ and $3.6 \mathrm{~Hz})$, $7.96(\mathrm{~d}, 1 \mathrm{H}, J=3.0 \mathrm{~Hz}), 9.13(\mathrm{bs}, 1 \mathrm{H}) .{ }^{13} \mathrm{C}-\mathrm{NMR}\left(\mathrm{CDCl}_{3}, 100 \mathrm{MHz}\right): \delta=51.8,108.8(\mathrm{~d}, J=23 \mathrm{~Hz}), 109.4$ $(\mathrm{d}, J=3.5 \mathrm{~Hz}), 112.1(\mathrm{~d}, J=4.1 \mathrm{~Hz}), 115.1(\mathrm{~d}, J=22 \mathrm{~Hz}), 123.0(\mathrm{~d}, J=8.0 \mathrm{~Hz}), 132.2,135.6(\mathrm{~d}, J=10.0 \mathrm{~Hz})$, $155.1(\mathrm{~d}, J=253 \mathrm{~Hz}), 164.0 \mathrm{ppm}$. HRMS $\left(\mathrm{ESI}^{+}\right) \mathrm{m} / \mathrm{z}[\mathrm{M}+\mathrm{Na}]^{+}$calculated for $\mathrm{C}_{10} \mathrm{H}_{7} \mathrm{ClFNO}_{2} \mathrm{Na} 250.0047$, found 250.0051 .

Methyl 4,7-dibromo-1H-indole-3-carboxylate (6ab) $(598.0 \mathrm{mg}, 45 \%)$. Off-white solid: ${ }^{1} \mathrm{H}-\mathrm{NMR}$ (DMSO- $\left.d_{6}, 500 \mathrm{MHz}\right): \delta=3.78(\mathrm{~s}, 3 \mathrm{H}), 7.33(\mathrm{~d}, 1 \mathrm{H}, J=8.0 \mathrm{~Hz}), 7.37(\mathrm{~d}, 1 \mathrm{H}, J=8.0 \mathrm{~Hz}), 8.09(\mathrm{~d}, 1 \mathrm{H}$, $J=3.1 \mathrm{~Hz}), 12.40(\mathrm{bs}, 1 \mathrm{H}) .{ }^{13} \mathrm{C}-\mathrm{NMR}\left(\mathrm{DMSO}_{\mathrm{d}}, 125 \mathrm{MHz}\right): \delta=51.1,104.6,109.1,112.3,125.3,126.1$, 127.3, 134.3, 136.0, $163.3 \mathrm{ppm}$. HRMS $\left(\mathrm{ESI}^{+}\right) \mathrm{m} / \mathrm{z}[\mathrm{M}+\mathrm{Na}]^{+}$calculated for $\mathrm{C}_{10} \mathrm{H}_{7} \mathrm{Br}_{2} \mathrm{NO}_{2} \mathrm{Na} 357.8700$, found 357.8698 .

Methyl 1H-benzo[g]indole-3-carboxylate (6ac) $(745.8 \mathrm{mg}, 83 \%)$. Yellow-brown solid: ${ }^{1} \mathrm{H}-\mathrm{NMR}$ (DMSO-d $6,400 \mathrm{MHz}): \delta=3.85(\mathrm{~s}, 3 \mathrm{H}), 7.48(\mathrm{t}, 1 \mathrm{H}, J=7.5 \mathrm{~Hz}), 7.60(\mathrm{t}, 1 \mathrm{H}, J=7.5 \mathrm{~Hz}), 7.65(\mathrm{~d}, 1 \mathrm{H}$, $J=8.7 \mathrm{~Hz}), 7.97(\mathrm{~d}, 1 \mathrm{H}, J=8.2 \mathrm{~Hz}), 8.10-8.19(\mathrm{~m}, 2 \mathrm{H}), 8.44(\mathrm{~d}, 1 \mathrm{H}, J=8.2 \mathrm{~Hz}), 12.84(\mathrm{~s}, 1 \mathrm{H}) .{ }^{13} \mathrm{C}-\mathrm{NMR}$ (DMSO- $d_{6}, 100 \mathrm{MHz}$ ): $\delta=51.6,109.1,121.0,121.6,122.8,122.9,122.9,125.3,126.8,129.3,130.9,130.9$, 132.1, 165.8. The values are in accordance with the literature [85].

(6-Methoxy-1H-indol-3-yl)(3,4,5-trimethoxyphenyl)methanone (6ad; C-6 isomer) (257.3 $\mathrm{mg}, 30 \%)$. Off-white solid: ${ }^{1} \mathrm{H}-\mathrm{NMR}\left(\mathrm{CDCl}_{3}, 500 \mathrm{MHz}\right): \delta=3.79(\mathrm{~s}, 3 \mathrm{H}), 3.83(\mathrm{~s}, 6 \mathrm{H}), 3.91(\mathrm{~s}, 3 \mathrm{H}), 6.87(\mathrm{~d}, 1 \mathrm{H}$, $J=2.2 \mathrm{~Hz}), 6.93(\mathrm{dd}, 1 \mathrm{H}, J=8.7$ and $2.2 \mathrm{~Hz}), 7.07(\mathrm{~s}, 2 \mathrm{H}), 7.59(\mathrm{~d}, 1 \mathrm{H}, J=3.0 \mathrm{~Hz}), 8.21(\mathrm{~d}, 1 \mathrm{H}, J=8.7 \mathrm{~Hz})$, 9.48 (bs, $1 \mathrm{H}) .{ }^{13} \mathrm{C}-\mathrm{NMR}\left(\mathrm{CDCl}_{3}, 125 \mathrm{MHz}\right): \delta=55.5,56.2(2 \mathrm{C}), 60.9,95.1,106.4(2 \mathrm{C}), 112.2,116.7,120.4$, $122.9,132.9,135.9,137.5,140.9,152.9(2 \mathrm{C}), 157.5,190.6 \mathrm{ppm}$. The values are in accordance with the literature [20].

(4-Methoxy-1H-indol-3-yl)(3,4,5-trimethoxyphenyl)methanone (6ad; C-4 isomer) (212.1 $\mathrm{mg}, 25 \%$ ). Off-white solid: ${ }^{1} \mathrm{H}-\mathrm{NMR}$ (DMSO-d $\left.\mathrm{D}_{6}, 500 \mathrm{MHz}\right): \delta=3.65(\mathrm{~s}, 3 \mathrm{H}), 3.75(\mathrm{~s}, 3 \mathrm{H}), 3.77(\mathrm{~s}, 6 \mathrm{H}), 6.63(\mathrm{~d}, 1 \mathrm{H}$, $J=7.8 \mathrm{~Hz}), 7.09(\mathrm{~s}, 2 \mathrm{H}), 7.10(\mathrm{~d}, 1 \mathrm{H}, J=7.8 \mathrm{~Hz}), 7.16(\mathrm{t}, 1 \mathrm{H}, J=7.9 \mathrm{~Hz}), 7.75(\mathrm{~d}, 1 \mathrm{H}, J=3.0 \mathrm{~Hz}), 11.86$ (bs, $1 \mathrm{H}) .{ }^{13} \mathrm{C}-\mathrm{NMR}$ (DMSO- $\left.d_{6}, 125 \mathrm{MHz}\right): \delta=55.0,55.9(2 \mathrm{C}), 60.1,101.9,105.2,107.0(2 \mathrm{C}), 115.7,115.9$, $123.7,131.6,135.3,138.2,140.7,152.3(2 \mathrm{C}), 153.6,188.3 \mathrm{ppm}$. The values are in accordance with the literature [20].

( \pm -Methyl (2R,3R,3aS)-2-hydroxy-3a-methyl-3,3a-dihydro-2H-indole-3-carboxylate (10s) (144.1 mg, 21\%). Off-white solid: ${ }^{1} \mathrm{H}-\mathrm{NMR}\left(\mathrm{CDCl}_{3}, 400 \mathrm{MHz}\right): \delta=1.00(\mathrm{~s}, 3 \mathrm{H}), 2.95(\mathrm{~d}, 1 \mathrm{H}, J=7.4 \mathrm{~Hz}), 3.79(\mathrm{~s}, 3 \mathrm{H})$, $5.7(\mathrm{bs}, 1 \mathrm{H}), 5.89(\mathrm{~d}, 1 \mathrm{H}, J=7.4 \mathrm{~Hz}), 6.09-6.13(\mathrm{~m}, 1 \mathrm{H}), 6.44-6.47(\mathrm{~m}, 2 \mathrm{H}), 6.58-6.62(\mathrm{~m}, 1 \mathrm{H}) .{ }^{13} \mathrm{C}-\mathrm{NMR}$ $\left(\mathrm{CDCl}_{3}, 100 \mathrm{MHz}\right): \delta=20.0,52.1,53.3,61.8,91.0,120.7,121.3,136.0,140.4,170.5,178.3$ ppm. HRMS $\left(\mathrm{ESI}^{+}\right) \mathrm{m} / \mathrm{z}[\mathrm{M}+\mathrm{Na}]^{+}$calculated for $\mathrm{C}_{11} \mathrm{H}_{13} \mathrm{NO}_{3} \mathrm{Na} 230.0793$, found 230.0785 .

( \pm )-(2R,3R,3aR)-Methyl 2-hydroxy-3a,4-dimethyl-3,3a-dihydro-2H-indole-3-carboxylate (10t) (150.6 mg, 34\%). Off-white solid: ${ }^{1} \mathrm{H}-\mathrm{NMR}\left(\mathrm{CDCl}_{3}, 500 \mathrm{MHz}\right): \delta=1.13(\mathrm{~s}, 3 \mathrm{H}), 1.91(\mathrm{~s}, 3 \mathrm{H}), 2.91(\mathrm{~d}, 1 \mathrm{H}, J=7.4 \mathrm{~Hz})$, $3.77(\mathrm{~s}, 3 \mathrm{H}), 5.4(\mathrm{bs}, 1 \mathrm{H}), 5.80(\mathrm{~d}, 1 \mathrm{H}, J=6.0 \mathrm{~Hz}), 5.85(\mathrm{~d}, 1 \mathrm{H}, J=7.4 \mathrm{~Hz}), 6.27(\mathrm{~d}, 1 \mathrm{H}, J=9.7 \mathrm{~Hz}), 6.50(\mathrm{dd}$, $1 \mathrm{H}, J=9.7$ and $6.0 \mathrm{~Hz}) .{ }^{13} \mathrm{C}-\mathrm{NMR}\left(\mathrm{CDCl}_{3}, 125 \mathrm{MHz}\right): \delta=15.4,20.1,52.0,57.9,60.5,94.1,118.4,118.6$, 136.5, 150.3, 171.6, $179.3 \mathrm{ppm}$. HRMS $\left(\mathrm{ESI}^{+}\right) \mathrm{m} / \mathrm{z}[\mathrm{M}+\mathrm{Na}]^{+}$calculated for $\mathrm{C}_{12} \mathrm{H}_{15} \mathrm{NO}_{3} \mathrm{Na} 244.0950$, found 244.0952 . 
(土)-(2R,3R,3aS)-Methyl 4-chloro-2-hydroxy-3a-methyl-3,3a-dihydro-2H-indole-3-carboxylate (10u) (128.8 mg, 27\%). Off-white solid: ${ }^{1} \mathrm{H}-\mathrm{NMR}\left(\mathrm{CDCl}_{3}, 500 \mathrm{MHz}\right): \delta=1.30(\mathrm{~s}, 3 \mathrm{H}), 3.02(\mathrm{~d}, 1 \mathrm{H}, \mathrm{J}=7.5 \mathrm{~Hz})$, $3.77(\mathrm{~s}, 3 \mathrm{H}), 5.7(\mathrm{bs}, 1 \mathrm{H}), 5.75(\mathrm{~d}, 1 \mathrm{H}, J=7.5 \mathrm{~Hz}), 6.16(\mathrm{~d}, 1 \mathrm{H}, J=6.3 \mathrm{~Hz}), 6.42(\mathrm{~d}, 1 \mathrm{H}, J=9.6 \mathrm{~Hz}), 6.55$ $(\mathrm{dd}, 1 \mathrm{H}, J=9.6$ and $6.3 \mathrm{~Hz}) .{ }^{13} \mathrm{C}-\mathrm{NMR}\left(\mathrm{CDCl}_{3}, 125 \mathrm{MHz}\right): \delta=19.8,52.2,59.9,61.0,93.8,119.5,120.4$, 135.5, 144.5, 170.8, 177.5 ppm. HRMS $\left(\mathrm{ESI}^{+}\right) \mathrm{m} / \mathrm{z}[\mathrm{M}+\mathrm{Na}]^{+}$calculated for $\mathrm{C}_{11} \mathrm{H}_{12} \mathrm{ClNO}_{3} 264.0403$, found 264.0404 .

The relative stereochemistry was assigned based on the ${ }^{1} \mathrm{HNMR}, \mathrm{COSY}, \mathrm{HSQC}$ and NOESY experiments. The syn relationship between $\mathrm{H}(2)$ and $\mathrm{H}(3)$ was assigned based on the observed ${ }^{3} J(\mathrm{H}, \mathrm{H})$ $=7.5 \mathrm{~Hz}$ and their NOE interactions. The syn relationship between $\mathrm{Me}(3 \mathrm{a})$ and $\mathrm{H}(3)$ was assigned based on their NOE interactions.

(土)-Methyl (2R,3R,3aR)-2-hydroxy-3a-methoxy-3,3a-dihydro-2H-indole-3-carboxylate (10z) (195.8 mg, 22\%). Off-white solid: ${ }^{1} \mathrm{H}-\mathrm{NMR}\left(\mathrm{CDCl}_{3}, 500 \mathrm{MHz}\right): \delta=2.87(\mathrm{~d}, 1 \mathrm{H}, J=6.1 \mathrm{~Hz}), 3.03(\mathrm{~s}, 3 \mathrm{H}), 3.79(\mathrm{~s}, 3 \mathrm{H})$, $3.9(\mathrm{bs}, 1 \mathrm{H}), 6.21(\mathrm{~d}, 1 \mathrm{H}, J=6.1 \mathrm{~Hz}), 6.43(\mathrm{~d}, 1 \mathrm{H}, J=9.6 \mathrm{~Hz}), 6.51(\mathrm{ddd}, 1 \mathrm{H}, J=9.6,5.5 \mathrm{and} 1.0 \mathrm{~Hz})$, 6.58-6.62 (m, 2H), $6.67(\mathrm{~d}, 1 \mathrm{H}, J=9.6 \mathrm{~Hz}) .{ }^{13} \mathrm{C}-\mathrm{NMR}\left(\mathrm{CDCl}_{3}, 125 \mathrm{MHz}\right): \delta=52.2,52.4,65.4,81.4,91.8$, $123.3,127.4,131.4,134.4,168.6,172.3 \mathrm{ppm}$. HRMS $\left(\mathrm{ESI}^{+}\right) \mathrm{m} / \mathrm{z}[\mathrm{M}+\mathrm{Na}]^{+}$calculated for $\mathrm{C}_{11} \mathrm{H}_{13} \mathrm{NO}_{4} \mathrm{Na}$ 246.0742 , found 246.0735 .

\section{Conclusions}

In summary, we reported a practical (scalable) and instrumentally simple methodology to synthesize $\mathrm{N}$-unprotected polysubstituted indoles endowed with an electron-withdrawing group at C-3 position. The importance of these N-unprotected heterocycles in organic synthesis (molecular platforms), asymmetric catalysis (chiral catalysts), medicinal chemistry (therapeutics) and materials sciences (molecular devices) ensures the practical use of this methodology in different fields of organic chemistry. Studies aimed at the use of these 3-EWG-indoles as molecular platforms for catalytic asymmetric dearomatization and their therapeutic/biological annotations are in progress in our lab.

Supplementary Materials: ${ }^{1} \mathrm{H}$ and ${ }^{13} \mathrm{C}-\mathrm{NMR}$ spectra for compounds $\mathbf{6}$ and $\mathbf{1 0}$ are available online.

Author Contributions: R.D.-R. and D.T. conducted experimental work; D.T. and F.G.-T. initiated the project, designed experiments and wrote the paper. All authors have read and agreed to the published version of the manuscript.

Funding: This research was funded by the Spanish Ministry of Science, Innovation and Universities (MICINN), State Research Agency (AEI) and the European Regional Development Funds (ERDF) (PGC2018-094503-B-C21).

Acknowledgments: Authors thank La Laguna University for the use of SEGAI.

Conflicts of Interest: The authors declare no conflict of interest.

\section{References and Note}

1. Barluenga, J.; Valdés, C. Five-membered heterocycles: Indole and related systems. In Modern Heterocyclic Chemistry; Alvarez-Builla, J., Vaquero, J.J., Barluenga, J., Eds.; Wiley-VCH Verlag GmbH \& Co.: Weinheim, Germany, 2011; pp. 377-531.

2. Dhuguru, J.; Skouta, R. Role of indole scafolds as pharmacophores in the development of anti-lung cancer agents. Molecules 2020, 25, 1615. [CrossRef]

3. Wan, Y.; Li, Y.; Yan, C.; Yan, M.; Tang, Z. Indole: A privileged scaffold for the design of anti-cancer agents. Eur. J. Med. Chem. 2019, 183, 111691-111709. [CrossRef] [PubMed]

4. Goyal, D.; Kaur, A.; Goyal, B. Benzofuran and indole: Promising scaffolds for drug development in Alzheimer's disease. ChemMedChem 2018, 13, 1275-1299. [CrossRef] [PubMed]

5. Chadha, N.; Silakari, O. Indoles as therapeutics of interest in medicinal chemistry: Bird's eye view. Eur. J. Med. Chem. 2017, 134, 159-184. [CrossRef] [PubMed]

6. Gribble, G.W. Indole Ring Synthesis: From Natural Products to Drug Discovery; John Wiley \& Sons: Chichester, UK, 2016. 
7. Owczarczyk, Z.R.; Braunecker, W.A.; Garcia, A.; Larsen, R.; Nardes, A.M.; Kopidakis, N.; Ginley, D.S.; Olson, D.C. 5,10-Dihydroindolo[3,2-b]indole-based copolymers with alternating donor and acceptor moieties for organic photovoltaics. Macromolecules 2013, 46, 1350-1360. [CrossRef]

8. Nie, G.M.; Bai, Z.M.; Yu, W.Y.; Zhang, L. Electrochemiluminescence biosensor for ramos cells based on a nanostructured conducting polymer composite material (PICA-MWNTs). J. Polym. Sci. Part A Polym. Chem. 2013, 51, 2385-2392. [CrossRef]

9. Manickam, M.; Iqbal, P.; Belloni, M.; Kumar, S.; Preece, J.A. A brief review of carbazole-based photorefractive liquid crystalline materials. Isr. J. Chem. 2012, 52, 917-934. [CrossRef]

10. Li, T.Z.; Liu, S.J.; Tan, W.; Shi, F. For a recent review on the current state of the field, see: Catalytic asymmetric construction of axially chiral indole-based frameworks: An emerging area. Chem. Eur. J. 2020, 26. [CrossRef]

11. Zhang, Y.C.; Jiang, F.; Shi, F. Organocatalytic asymmetric synthesis of indole-based chiral heterocycles: Strategies, reactions, and outreach. Acc. Chem. Res. 2020, 53, 425-446. [CrossRef]

12. Ma, C.; Sheng, F.T.; Wang, H.Q.; Deng, S.; Zhang, Y.C.; Jiao, Y.; Tan, W.; Shi, F. Atroposelective access to oxindole-based axially chiral styrenes via the strategy of catalytic kinetic resolution. J. Am. Chem. Soc. 2020, 142, 15686-15696. [CrossRef]

13. Lai, Y.T.; Wang, T.; O’Dell, S.; Louder, M.K.; Schön, A.; Cheung, C.S.; Chuang, G.Y.; Druz, A.; Lin, B.; McKee, K.; et al. Lattice engineering enables definition of molecular features allowing for potent small-molecule inhibition of HIV-1 entry. Nat. Commun. 2019, 10, 47-58. [CrossRef] [PubMed]

14. Liu, T.; Huang, B.; Zhan, P.; De Clercq, E.; Liu, X. Discovery of small molecular inhibitors targeting HIV-1 gp120eCD4 interaction drived from BMS-378806. J. Eur. Med. Chem. 2014, 86, 481-490. [CrossRef] [PubMed]

15. Lei, H.; Guo, M.; Li, X.; Jia, F.; Li, C.; Yang, Y.; Cao, M.; Jiang, N.; Ma, E.; Zhai, X.; et al. Discovery of novel indole-based allosteric highly potent ATX inhibitors with great in vivo efficacy in a mouse lung fibrosis model. J. Med. Chem. 2020, 63, 7326-7346. [CrossRef] [PubMed]

16. Apirakkan, O.; Gavrilović, I.; Cowan, D.A.; Abbate, V. In vitro phase i metabolic profiling of the synthetic cannabinoids AM-694, 5F-NNEI, FUB-APINACA, MFUBINAC, and AMB-FUBINACA. Chem. Res. Toxicol. 2020, 33, 1653-1664. [CrossRef]

17. Liou, J.P. 3-Aroylindoles display antitumor activity in vitro and in vivo: Effects of N1-substituents on biological activity. J. Med. Chem. 2017, 125, 1268-1278.

18. Smith, A.C.; Kung, D.W.; Shavnya, A.; Brandt, T.A.; Dent, P.D.; Genung, N.E.; Cabral, S.; Panteleev, J.; Herr, M.; Yip, K.N.; et al. Evolution of the synthesis of AMPK activators for the treatment of diabetic nephropathy: From three preclinical candidates to the investigational new drug PF-06409577. Org. Process. Res. Dev. 2018, 22, 681-696. [CrossRef]

19. Wu, Y.S.; Coumar, M.S.; Chang, J.Y.; Sun, H.Y.; Kuo, F.M.; Kuo, C.C.; Chen, Y.J.; Chang, C.Y.; Hsiao, C.L.; Liou, J.P.; et al. Synthesis and evaluation of 3-aroylindoles as anticancer agents: Metabolite approach. J. Med. Chem. 2009, 52, 4941-4945. [CrossRef]

20. Liou, J.P.; Chang, Y.L.; Kuo, F.M.; Chang, C.W.; Tseng, H.Y.; Wang, C.C.; Yang, Y.N.; Chang, J.Y.; Lee, S.J.; Hsieh, H.P.; et al. Concise synthesis and structure-activity relationships of combretastatin A-4 analogues, 1-aroylindoles and 3-aroylindoles, as novel classes of potent antitubulin agents. J. Med. Chem. 2004, 47, 4247-4257. [CrossRef]

21. Kuo, C.C.; Hsieh, H.P.; Pan, W.Y.; Chen, C.P.; Liou, J.P.; Lee, S.J.; Chang, Y.L.; Chen, L.T.; Chen, C.T.; Chang, J.Y.; et al. BPR0L075, a novel synthetic indole compound with antimitotic activity in human cancer cells, exerts effective antitumoral activity in vivo. Cancer Res. 2004, 64, 4621-4628. [CrossRef]

22. Huck, C.J.; Sarlah, D. Shaping Molecular Landscapes: Recent Advances, Opportunities, and Challenges in Dearomatization. Chem 2020, 6, 1589-1603. [CrossRef]

23. Li, K.; Gonçalves, T.P.; Huang, K.W.; Lu, Y. Dearomatization of 3-nitroindoles by a phosphine-catalyzed enantioselective [3 + 2] annulation reaction. Angew. Chem. Int. Ed. 2019, 58, 5427-5431. [CrossRef] [PubMed]

24. Wang, H.; Zhang, J.; Tu, Y.; Zhang, J. Phosphine-catalyzed enantioselective dearomative [3 + 2]-cycloaddition of 3-nitroindoles and 2-nitrobenzofurans. Angew. Chem. Int. Ed. 2019, 58, 5422-5426. [CrossRef] [PubMed]

25. Suo, J.J.; Liu, W.; Du, J.; Ding, C.H.; Hou, X.L. Diastereo-and enantioselective palladium-catalyzed dearomative [3 + 2] cycloaddition of 3-nitroindoles. Chem. Asian J. 2018, 13, 959-963. [CrossRef] [PubMed]

26. Cheng, Q.; Zhang, F.; Cai, Y.; Guo, Y.L.; You, S.L. Stereodivergent synthesis of tetrahydrofuroindoles through Pd-catalyzed asymmetric dearomative formal [3 +2] cycloaddition. Angew. Chem. Int. Ed. 2018, 57, 2134-2138. [CrossRef] [PubMed] 
27. Sun, M.; Zhu, Z.Q.; Gu, L.; Wan, X.; Mei, G.J.; Shi, F. Catalytic asymmetric dearomative [3 + 2] cycloaddition of electron-deficient indoles with all-carbon 1,3-dipoles. J. Org. Chem. 2018, 83, 2341-2348. [CrossRef]

28. Zhang, J.Q.; Tong, F.; Sun, B.B.; Fan, W.T.; Chen, J.B.; Hu, D.; Wang, X.W. Pd-catalyzed asymmetric dearomative cycloaddition for construction of optically active pyrroloindoline and cyclopentaindoline derivatives: Access to 3a-aminopyrroloindolines. J. Org. Chem. 2018, 83, 2882-2891. [CrossRef]

29. Yue, D.F.; Zhao, J.Q.; Chen, X.Z.; Zhou, Y.; Zhang, X.M.; Xu, X.Y.; Yuan, W.C. Multiple hydrogen-bonding bifunctional thiourea-catalyzed asymmetric dearomative [4 +2$]$ annulation of 3-nitroindoles: Highly enantioselective access to hydrocarbazole skeletons. Org. Lett. 2017, 19, 4508-4511. [CrossRef]

30. Gerten, L.; Stanley, L.M. Enantioselective dearomative [3 + 2] cycloadditions of indoles with azomethine ylides derived from alanine imino esters. Org. Chem. Front. 2016, 3, 339-343. [CrossRef]

31. Zhao, J.Q.; Wu, Z.J.; Zhou, M.Q.; Xu, X.Y.; Zhang, X.M.; Yuan, W.C. Zn-catalyzed diastereo-and enantioselective cascade reaction of 3-isothiocyanato oxindoles and 3-nitroindoles: Stereocontrolled syntheses of polycyclic spirooxindoles. Org. Lett. 2015, 17, 5020-5023. [CrossRef]

32. Awata, A.; Arai, T. PyBidine/Copper catalyst: Asymmetric exo'-selective [3 + 2] cycloaddition using imino ester and electrophilic indole. Angew. Chem. Int. Ed. 2014, 53, 10462-10465. [CrossRef]

33. Li, Y.; Tur, F.; Nielsen, R.P.; Jiang, H.; Jensen, F.; Jørgensen, K.A. Enantioselective formal [4 + 2] cycloadditions to 3-nitroindoles by trienamine catalysis: Synthesis of chiral dihydrocarbazoles. Angew. Chem. Int. Ed. 2016, 55, 1020-1024. [CrossRef] [PubMed]

34. Roche, S.P.; Tendoung, J.J.Y.; Treguier, B. Advances in dearomatization strategies of indoles. Tetrahedron 2015, 71, 3549-3591. [CrossRef]

35. Kumar, N.; Parle, A. 3-Substituted indole: A review. Int. J. Chem. Stud. 2019, 72, 848-856.

36. Norwood, V.M.; Huigens, R.W. Harnessing the chemistry of the indole heterocycle to drive discoveries in biology and medicine. ChemBioChem 2019, 20, 2273-2297. [CrossRef]

37. Joule, J.A. Indole and its derivatives. Chapter 10.13. In Science of Synthesis: Houben-Weyl Methods of Molecular Transformations; Thomas, E.J., Ed.; George Thieme Verlag: Stuttgart, Germany, 2000; Volume 10, pp. 361-652.

38. Li, L.H.; Niu, Z.J.; Liang, Y.M. New Friedel-Crafts strategy for preparing 3-acylindoles. Org. Biomol. Chem. 2018, 16, 7792-7796. [CrossRef]

39. Wu, W.; Su, W. Mild and selective Ru-catalyzed formylation and Fe-catalyzed acylation of free (NH) indoles using anilines as the carbonyl source. J. Am. Chem. Soc. 2011, 133, 11924-11927. [CrossRef]

40. Guchhait, S.K.; Kashyap, M.; Kamble, H. $\mathrm{ZrCl}_{4}$-Mediated regio-and chemoselective friedel-crafts acylation of indole. J. Org. Chem. 2011, 76, 4753-4758. [CrossRef]

41. Katritzky, R.; Suzuki, K.; Singh, S.K.; He, H.Y. Regiospecific C-acylation of pyrroles and indoles using N-acylbenzotriazoles. J. Org. Chem. 2003, 58, 5720-5723. [CrossRef]

42. Okauchi, T.; Itonaga, M.; Minami, T.; Owa, T.; Kitoh, K.; Yoshino, H. A general method for acylation of indoles at the 3-position with acyl chlorides in the presence of dialkylaluminum chloride. Org. Lett. 2000, 2, 1485-1487. [CrossRef]

43. Zhang, W.; Tang, J.; Yu, W.; Huang, Q.; Fu, Y.; Kuang, G.; Pan, C.; Yu, G. Visible light-driven C-3 functionalization of indoles over conjugated microporous polymers. ACS Catal. 2018, 8, 8084-8091. [CrossRef]

44. Wu, C.J.; Meng, Q.Y.; Lei, T.; Zhong, J.J.; Liu, W.Q.; Zhao, L.M.; Li, Z.J.; Chen, B.; Tung, C.H.; Wu, L.Z.; et al. An oxidant-free strategy for indole synthesis via intramolecular $\mathrm{C}-\mathrm{C}$ bond construction under visible light irradiation: Cross-coupling hydrogen evolution reaction. ACS Catal. 2016, 6, 4635-4639. [CrossRef]

45. Li, X.; Gu, X.; Li, Y.; Li, P. Aerobic transition-metal-free visible-light photoredox indole C-3 formylation reaction. ACS Catal. 2014, 4, 1897-1900. [CrossRef]

46. Zhang, P.; Xiao, T.; Xiong, S.; Dong, X.; Zhou, L. Synthesis of 3-acylindoles by visible-light induced intramolecular oxidative cyclization of o-alkynylated $N, N$-dialkylamines. Org. Lett. 2014, 16, 3264-3267. [CrossRef] [PubMed]

47. Humphrey, G.R.; Kuethe, J.T. Practical methodologies for the synthesis of indoles. Chem. Rev. 2006, 106, 2875-2911. [CrossRef]

48. Neto, J.S.S.; Zeni, G. Recent advances in the synthesis of indoles from alkynes and nitrogen sources. Org. Chem. Front. 2020, 7, 155-210. [CrossRef]

49. Inman, M.; Moody, C.J. Indole synthesis-something old, something new. Chem. Sci. 2013, 4, 29-41. [CrossRef]

50. Tabolin, A.A.; Ioffe, S.L. Rearrangement of N-oxyenamines and related reactions. Chem. Rev. 2014, 114, 5426-5476. [CrossRef] 
51. Heravi, M.M.; Rohani, S.; Zadsirjan, V.; Zahedi, N. Fischer indole synthesis applied to the total synthesis of natural products. RSC Adv. 2017, 7, 52852-52887. [CrossRef]

52. Hughes, D.L. Progress in the Fischer indole reaction. A review. Org. Prep. Proced. Int. 1993, 25, 607-632. [CrossRef]

53. Bartoli, G.; Dalpozzo, R.; Nardib, M. Applications of Bartoli indole synthesis. Chem. Soc. Rev. 2014, 43, 4728-4750. [CrossRef]

54. Dalpozzo, R.; Bartoli, G. Bartoli indole synthesis. Curr. Org. Chem. 2005, 9, 163-178. [CrossRef]

55. Fischer, E.E.; Jourdan, F. Ueber die hydrazine der brenztraubensäure. Eur. J. Inorg. Chem. 1883, 16, 2241-2245. [CrossRef]

56. Wang, Y.; Liu, L.; Zhang, L. Combining Zn ion catalysis with homogeneous gold catalysis: An efficient annulation approach to N-protected indoles. Chem. Sci. 2013, 4, 739-746. [CrossRef] [PubMed]

57. Yuan, H.; Guo, L.; Liu, F.; Miao, Z.; Feng, L.; Gao, H. A copper-catalyzed synthesis of polysubstituted indoles using $\mathrm{N}$-arylhydroxylamines and vinyliodonium salts using the same disconnection pattern has been recently described, although its application to 3-EWG-indoles was not reported. Copper-catalyzed tandem o-vinylation of arylhydroxylamines/[3,3]-rearrangement/cyclization: Synthesis of highly substituted indoles and benzoindoles. ACS Catal. 2019, 9, 3906-3912.

58. Tejedor, D.; Delgado-Hernández, S.; Colella, L.; García-Tellado, F. Catalytic hydrocyanation of activated terminal alkynes. Chem. Eur. J. 2019, 25, 15046-15049. [CrossRef]

59. Tejedor, D.; Álvarez-Méndez, S.J.; López-Soria, J.M.; Martín, V.S.; García-Tellado, F. A robust and general protocol for the Lewis-base-catalysed reaction of alcohols and alkyl propiolates. Eur. J. Org. Chem. 2014, 198-205. [CrossRef]

60. Mola, L.; Font, J.; Bosch, L.; Caner, J.; Costa, A.M.; Etxebarría-Jardí, G.; Pineda, O.; De Vicente, D.; Vilarrasa, J. Nucleophile-catalyzed additions to activated triple bonds. Protection of lactams, imides, and nucleosides with MocVinyl and related groups. J. Org. Chem. 2013, 78, 5832-5842. [CrossRef]

61. Tejedor, D.; Santos-Expósito, A.; Méndez-Abt, G.; Ruiz-Pérez, C.; García-Tellado, F. Trialkylamine versus trialkylphosphine: Catalytic conjugate addition of alcohols to alkyl propiolates. Synlett 2009, 1223-1226. [CrossRef]

62. Feierfeil, J.; Magauer, T. De novo synthesis of benzannelated heterocycles. Chem. Eur. J. 2018, 24, 1455-1458. [CrossRef]

63. Pereira, M.M.A.; Prabhakar, S.; Lobo, A.M. A Synthesis of the amaryllidaceae alkaloid pratosine. J. Nat. Prod. 1996, 59, 744-747. [CrossRef]

64. Hwu, J.R.; Patel, H.V.; Lin, R.J.; Gray, M.O. Novel methods for the synthesis of functionalized indoles from arylhydroxylamines and activated acetylenes. J. Org. Chem. 1994, 59, 1577-1582. [CrossRef]

65. Toyota, M.; Fukumoto, K. Tandem Michael addition-[3,3]sigmatropic rearrangement processes. Part 2. Construction of cyclopropa[3,4]pyrrolo [3,2-e]indol-4-one (CPI) unit of antitumour antibiotic CC-1065. J. Chem. Soc. Perkin Trans. 1992, 1, 547-552. [CrossRef]

66. Blechert, S. Hetero-Cope-rearrangements, region-controlled synthesis of indoles. Helv. Chim. Acta 1985, 68, 1835-1843. [CrossRef]

67. Chehardoli, G.; Bahmani, A. Synthetic strategies, SAR studies, and computer modeling of indole 2 and 3-carboxamides as the strong enzyme inhibitors: A review. Mol. Divers. 2020, 1-16. [CrossRef]

68. Grandberg, I.; Belyaeva, L.D.; Dmitriev, L.B. Ratio of indole isomers formed during the fischer cyclization of meta-substituted diethyl ketone phenylhydrazones. Chem. Het. Compd. 1971, 7, 1131. [CrossRef]

69. Krenske, E.H.; Burns, J.M.; McGeary, R.P. Claisen rearrangements of benzyl vinyl ethers: Theoretical investigation of mechanism, substituent effects, and regioselectivity. Org. Biomol. Chem. 2017, 15, 7887-7893. [CrossRef]

70. An ortho-methyl group dropped the yield of the reaction to $25 \%$.

71. Lee, K.N.; Lei, Z.; Morales-Rivera, C.A.; Liu, P.; Ngai, M.Y. Mechanistic studies on intramolecular C-H trifluoromethoxylation of (hetero)arenes via $\mathrm{OCF}_{3}$-migration. Org. Biomol. Chem. 2016, 14, 5599. [CrossRef]

72. Yang, Q.Q.; Marchini, M.; Xiao, W.J.; Ceroni, P.; Bandini, M. Visible-light-induced direct photocatalytic carboxylation of indoles with $\mathrm{CBr}_{4} / \mathrm{MeOH}$. Chem. Eur. J. 2015, 50, 18052-18056. [CrossRef]

73. Abe, T.; Yamada, K. Amination/cyclization cascade by acid-catalyzed activation of indolenine for the one-pot synthesis of phaitanthrin E. Org. Lett. 2016, 18, 6504-6507. [CrossRef] 
74. Yu, J.; Zhang, C.; Yang, X.; Su, W. Decarboxylative acylation of N-free indoles enabled by a catalytic amount of copper catalyst and liquid-assisted grinding. Org. Biomol. Chem. 2019, 17, 4446-4451. [CrossRef]

75. Wynne, J.H.; Lloyd, C.T.; Jensen, S.D.; Boson, S.; Stalick, W.M. 3-Acylindoles via a one-pot, regioselective Friedel-Crafts reaction. Synthesis 2004, 2277-2282. [CrossRef]

76. Peng, J.; Liu, L.; Hu, Z.; Huang, J.; Zhu, Q. Direct carboxamidation of indoles by palladium-catalyzed C-H activation and isocyanide insertion. Chem. Commun. 2012, 48, 3772-3774. [CrossRef] [PubMed]

77. Velavan, A.; Sumathi, S.; Balasubramanian, K.K. AlMe $\mathrm{A}_{3}$-mediated region- and chemoselective reactions of indole with carbamoyl electrophiles. Eur. J. Org. Chem. 2013, 3148-3157. [CrossRef]

78. Yamazaki, K.; Nakamura, Y.; Kondo, Y. Solid-phase synthesis of indolecarboxylates using palladium-catalyzed reactions. J. Org. Chem. 2003, 68, 6011-6019. [CrossRef]

79. Linton, E.C.; Kozlowski, M.C. Catalytic enantioselective Meerwein-Eschenmoser Claisen rearrangement: Asymmetric synthesis of allyl oxindoles. J. Am. Chem. Soc. 2008, 130, 16162-16163. [CrossRef]

80. Fisher, M.J.; McMurray, L.; Lu, S.; Morse, C.L.; Liow, J.S.; Zoghbi, S.S.; Kowalski, A.; Tye, G.L.; Innis, R.B.; Aigbirhio, F.I.; et al. [Carboxyl-11 C]labelling of four high-affinity cPLA2 $\alpha$ inhibitors and their evaluation as radioligands in mice by positron emission tomography. ChemMedChem 2018, 13, 138-146. [CrossRef]

81. Natarajan, R.; Rappa, J.P.; Unnikrishnan, P.A.; Radhaman, S.; Prathapan, S. A new method for the synthesis of 3-substituted indoles. Synlett 2015, 26, 2467-2471.

82. Zhang, Z.W.; Xue, H.; Li, H.; Kang, H.; Feng, J.; Lin, A.; Liu, S. Collective synthesis of 3-acylindoles, indole-3-carboxylic esters, indole-3-sulfinic acids, and 3-(methylsulfonyl)indoles from free (N-H) indoles via common N-indolyl triethylborate. Org. Lett. 2016, 18, 3918-3921. [CrossRef]

83. Ji, X.; Huang, H.; Wu, W.; Li, X.; Jiang, H. Palladium-catalyzed oxidative coupling of aromatic primary amines and alkenes under molecular oxygen: Stereoselective assembly of (Z)-enamines. J. Org. Chem. 2013, 38, 11155-11162. [CrossRef]

84. Rashad, M.; La Vecchia, L.; Prasad, K.; Repic, O. A convenient synthesis of 3-substituted 1H-indoles. Synth. Commun. 1995, 25, 95-100. [CrossRef]

85. Yoo, W.J.; Capdevila, M.G.; Du, X.; Kobayashi, S. Base-mediated carboxylation of unprotected indole derivatives with carbon dioxide. Org. Lett. 2012, 14, 5326-5329. [CrossRef] [PubMed]

Sample Availability: Samples of the compounds 6a-ad are available from the authors.

Publisher's Note: MDPI stays neutral with regard to jurisdictional claims in published maps and institutional affiliations.

(C) 2020 by the authors. Licensee MDPI, Basel, Switzerland. This article is an open access article distributed under the terms and conditions of the Creative Commons Attribution (CC BY) license (http://creativecommons.org/licenses/by/4.0/). 